RUNHETC-2003-08

\title{
Two-point correlation functions of scaling fields in the Dirac theory on the Poincaré disk
}

\author{
Benjamin Doyon \\ NHETC, Department of Physics and Astronomy \\ Rutgers University \\ Piscataway, NJ 08854, USA
}

\begin{abstract}
A result from Palmer, Beatty and Tracy suggests that the two-point function of certain spinless scaling fields in a free Dirac theory on the Poincaré disk can be described in terms of Painlevé VI transcendents. We complete and verify this description by fixing the integration constants in the Painlevé VI transcendent describing the two-point function, and by calculating directly in a Dirac theory on the Poincaré disk the long distance expansion of this two-point function and the relative normalization of its long and short distance asymptotics. The long distance expansion is obtained by developing the curved-space analogue of a form factor expansion, and the relative normalization is obtained by calculating the one-point function of the scaling fields in question. The long distance expansion in fact provides part of the solution to the connection problem associated with the Painlevé VI equation involved. Calculations are done using the formalism of angular quantization.
\end{abstract}

April 2003 


\section{Introduction}

Quantum Field Theory (QFT) in curved space-time is a subject of great interest which has been studied from many viewpoints (see for instance [1]). The main goal of QFT is the reconstruction of a set of correlation functions in which is embedded the physical information provided by the theory. Such a reconstruction is an important problem for QFT on curved space-time, where the structure of the theory is more subtle. A simple but non-trivial curved space-time with Euclidean signature is the Poincaré disk. It is maximally symmetric, which allows some techniques on two-dimensional flat space to be generalized to this space, and has a negative Gaussian curvature. The effect of a negative curvature is interesting to study: as was argued in [10, a negative curvature can be used as an infrared regulator for Euclidean QFT.

In two-dimensional flat space, a class of non-trivial correlation functions can be studied by their relation to the problem of isomonodromic deformations [2]. Correlators of certain scaling fields in a free massive Dirac theory are tau functions [2, 3, 4, 5, for the isomonodromic deformation problem associated to some Painlevé equations: they can be expressed in terms of Painlevé transcendents. The scaling fields in question, $\mathcal{O}_{\alpha}=\mathcal{O}_{-\alpha}^{\dagger},-1<\alpha<1$, are spinless, $U(1)$-neutral and have scaling dimension $\alpha^{2}$. They are not mutually local with respect to the Dirac field. Their mutual locality index with the Dirac field is $\alpha$, that is, the Dirac field $\Psi$ takes a factor, $\Psi \rightarrow e^{2 \pi i \alpha} \Psi$, when continued counterclockwise around the field $\mathcal{O}_{\alpha}$. Their physical interest stems in particular from the fact that correlators of some of these scaling fields are simply related to the scaling limit of correlators of local variables in the Ising model [6, 7, 8].

In [9], the authors generalized the method of isomonodromic deformation to the study of determinants of the Dirac operator on the Poincare disk. Their results suggest that the two-point function of scaling fields $\mathcal{O}_{\alpha}$ in a Dirac theory on the Poincaré disk can be expressed in terms of Painlevé VI transcendents. The general form of the Painlevé VI differential equation is:

$$
\begin{aligned}
& w^{\prime \prime}-\frac{1}{2}\left(\frac{1}{w}+\frac{1}{w-1}+\frac{1}{w-s}\right)\left(w^{\prime}\right)^{2}+\left(\frac{1}{s}+\frac{1}{s-1}+\frac{1}{w-s}\right) w^{\prime} \\
& =\frac{w(w-1)(w-s)}{s^{2}(1-s)^{2}}\left(\frac{\left(1-4 \mu^{2}\right) s(s-1)}{2(w-s)^{2}}-\frac{(\tilde{\lambda}-1)^{2} s}{2 w^{2}}+\frac{\gamma(s-1)}{(w-1)^{2}}+\frac{\lambda^{2}}{2}\right)
\end{aligned}
$$

where $w=w(s)$ and the primes mean derivatives with respect to $s$. There are four parameters: $\mu, \gamma, \lambda, \tilde{\lambda}$. For the description of the two-point function $\left\langle\mathcal{O}_{\alpha}(x) \mathcal{O}_{\alpha^{\prime}}(y)\right\rangle$ in the free Dirac theory on the Poincaré disk, with fermion mass $m$ and Gaussian curvature $-\frac{1}{R^{2}}$, the parameters are fixed to $\mu=m R, \lambda=\alpha-\alpha^{\prime}$, $\tilde{\lambda}=\alpha+\alpha^{\prime}$ and $\gamma=0$. The two-point function is then identified with the associated tau function $\tau(s)$, with $s$ simply related to the geodesic distance $\mathrm{d}(x, y)$ between the points $x$ and $y$ :

$$
\left\langle\mathcal{O}_{\alpha}(x) \mathcal{O}_{\alpha^{\prime}}(y)\right\rangle=\tau(s), \quad s=\tanh ^{2}\left(\frac{\mathrm{d}(x, y)}{2 R}\right) .
$$

Up to normalization, the tau function is given by [9]:

$$
\begin{aligned}
\frac{d}{d s} \ln \tau(s)= & \frac{s(1-s)}{4 w(1-w)(w-s)}\left(w^{\prime}-\frac{1-w}{1-s}\right)^{2}-\frac{\mu^{2}}{w-s} \\
& +\frac{\tilde{\lambda}^{2}}{4(1-s) w}+\frac{\lambda^{2} w}{4 s(1-s)}-\frac{\lambda^{2}}{4 s}+\frac{4 \mu^{2}-\tilde{\lambda}^{2}-\lambda^{2}}{4(1-s)} .
\end{aligned}
$$

This description for the correlator needs to be completed and to be compared with direct calculation in the Dirac theory on the Poincaré disk. In order to complete it, one must supply appropriate integration constants specifying the Painlevé transcendent that describes the correlator. First, the exponent $2 \alpha \alpha^{\prime}$ in the short distance power law of the two-point function,

$$
\left\langle\mathcal{O}_{\alpha}(x) \mathcal{O}_{\alpha^{\prime}}(y)\right\rangle \sim\left\langle\mathcal{O}_{\alpha+\alpha^{\prime}}\right\rangle \mathrm{d}(x, y)^{2 \alpha \alpha^{\prime}} \text { as } x \rightarrow y,
$$


specifies the exponent in the asymptotic behavior of $w(s)$ near the critical point $s=0$ :

$$
w \sim B s^{\alpha+\alpha^{\prime}} \text { as } s \rightarrow 0,
$$

where $B$ is some constant and where we must have $0<\alpha+\alpha^{\prime}<1$. Second, the cluster property of the two-point function,

$$
\left\langle\mathcal{O}_{\alpha}(x) \mathcal{O}_{\alpha^{\prime}}(y)\right\rangle=\left\langle\mathcal{O}_{\alpha}\right\rangle\left\langle\mathcal{O}_{\alpha^{\prime}}\right\rangle F\left(\frac{\mathrm{d}(x, y)}{2 R}\right), \quad \lim _{t \rightarrow \infty} F(t)=1
$$

specifies, for $\mu>\frac{1}{2}$, the exponent in the asymptotic behavior of $w(s)$ near the critical point $s=1$ :

$$
1-w \sim A(1-s)^{1+2 \mu} \text { as } s \rightarrow 1,
$$

where again, $A$ is some constant.

One can expect that the exponents in (1.4) and (1.6) form a set of integration constants fixing the Painlevé transcendent. However, one doesn't know a priori that there exists a solution to the Painlevé equation with both behaviors (1.4) and (1.6). In addition, even if such a solution exists, this set is not the most convenient. One cannot use it for instance to provide initial conditions for numerically solving the differential equation (1.1). It is more appropriate to fix the full expansion of the Painlevé transcendent near the singular point $s=1$, that is, to specify the constant $A$ in (1.6). Fixing this constant is part of the solution to the connection problem for the particular Painlevé VI equation that we are considering, that is, the problem of relating the behaviors of the Painlevé transcendent near its various critical points. The expansion of the Painlevé transcendent near $s=1$ is directly related to the long distance expansion of the two-point function:

$$
F(t)=1-A \frac{(\mu+\alpha)\left(\mu+\alpha^{\prime}\right)}{(2 \mu+1)^{2}} 4^{2 \mu+1} e^{-(4 \mu+2) t}+O\left(e^{-(4 \mu+4) t}\right) .
$$

To our knowledge, the theory of Painlevé VI equations (1.1) in the case $\gamma=0$ provides no expression for $A$ in terms of the exponents in (1.4) and (1.6). We calculated $A$ by evaluating directly in a free massive Dirac theory on the Poincaré disk the long distance expansion of the two-point function:

$$
A=\frac{\sin (\pi \alpha) \sin \left(\pi \alpha^{\prime}\right) \Gamma(\mu+\alpha) \Gamma(1+\mu-\alpha) \Gamma\left(\mu+\alpha^{\prime}\right) \Gamma\left(1+\mu-\alpha^{\prime}\right)}{\pi^{2} \Gamma(1+2 \mu)^{2}}
$$

The asymptotics (1.4) can also serve as initial condition once $B$ is known, although it is numerically not as efficient. From results by Jimbo concerning Painlevé VI equations [17, one can calculate the constant $B$ (see section 7 ) - this is another part of the solution to the connection problem.

Another important quantity concerning the two-point function that is not provided by the theory of Painlevé VI equations is an expression relating the normalization of the leading long distance asymptotics of the two-point function to that of its leading short distance asymptotics. Comparing (1.3) and (1.5), one sees that the relation between normalizations can be obtained by calculating the one-point function $\left\langle\mathcal{O}_{\alpha}\right\rangle$. Notice that the condition (1.3) fixes the normalization of the scaling fields $\mathcal{O}_{\alpha}$. This normalization being fixed, the one-point function $\left\langle\mathcal{O}_{\alpha}\right\rangle$ is unambiguous. By a simple generalization of calculations done on flat space in [11, 12], we found

$$
\left\langle\mathcal{O}_{\alpha}\right\rangle=(2 R)^{-\alpha^{2}} \prod_{n=1}^{\infty}\left(\frac{1-\frac{\alpha^{2}}{(\mu+n)^{2}}}{1-\frac{\alpha^{2}}{n^{2}}}\right)^{n} .
$$

The long distance asymptotics of the two-point function is obtained by a "form factor" expansion, that is, an expansion obtained by inserting between the two operators in an appropriate vacuum expectation value a resolution of the identity on an appropriate Hilbert space. Actual calculations of form factors on this Hilbert space are done in the formalism of angular quantization. More precisely, matrix elements of 
operators corresponding to the scaling fields $\mathcal{O}_{\alpha}$ between the vacuum and an excited state are evaluated through appropriate traces on the Hilbert space of angular quantization, following ideas from [13. 14, 15. The one-point function $\left\langle\mathcal{O}_{\alpha}\right\rangle$ is similarly evaluated through appropriate traces on the Hilbert space of angular quantization.

The plan of the paper is as follows. In section 2 we briefly recall standard results concerning the free Dirac fermion on the Poincaré disk. In section 3 we express the long distance expansion of the two-point function as a form factor expansion using canonical quantization. In section 4 we evaluate, up to normalization, the form factors of the scaling fields $\mathcal{O}_{\alpha}$ by calculating appropriate traces on the Hilbert space of angular quantization, and in section 5 we compute the one-point function $\left\langle\mathcal{O}_{\alpha}\right\rangle$, which specifies the normalization of the form factors. In section 6 we discuss the long distance expansion of the two-point function, and finally in section 7 we briefly elaborate on the description of 9 for the two-point function in terms of a Painlevé VI transcendent and clarify our results in this context.

\section{Free Dirac fermion on the Poincaré disk}

With appropriate complex coordinates $z=\mathrm{x}+i \mathrm{y}, \bar{z}=\mathrm{x}-i \mathrm{y}$, the Poincaré disk can be brought to the region $|z|<1$ in the complex $z$-plane. For a Gaussian curvature $-\frac{1}{R^{2}}$ the metric is then specified by

$$
d s^{2}=\frac{d z d \bar{z}(2 R)^{2}}{\left(1-|z|^{2}\right)^{2}}
$$

The metric is $S U(1,1)$ invariant. The transformation of coordinates corresponding to the group element $g=\left(\begin{array}{cc}a & b \\ \bar{b} & \bar{a}\end{array}\right),|a|^{2}-|b|^{2}=1$ is

$$
z \mapsto \frac{a z+\bar{b}}{b z+\bar{a}}, \bar{z} \mapsto \frac{\bar{a} \bar{z}+b}{\bar{b} \bar{z}+a}
$$

The geodesic distance $\mathrm{d}\left(x_{1}, x_{2}\right)$ between points $x_{1}=\left(z_{1}, \bar{z}_{1}\right)$ and $x_{2}=\left(z_{2}, \bar{z}_{2}\right)$ on the Poincaré disk is given by

$$
\mathrm{d}\left(x_{1}, x_{2}\right)=2 R \operatorname{arctanh}\left(\frac{\left|z_{1}-z_{2}\right|}{\left|1-z_{1} \bar{z}_{2}\right|}\right) .
$$

The free massive Dirac action with fermion mass $m$ in this system of coordinates is

$$
\mathcal{A}=\int d \mathrm{x} d \mathrm{y} \bar{\Psi}\left(\gamma^{\mathrm{x}} \partial_{\mathrm{x}}+\gamma^{\mathrm{y}} \partial_{\mathrm{y}}+\frac{2 \mu}{1-|z|^{2}}\right) \Psi
$$

where as in the introduction $\mu=m R$, and $\Psi=\left(\begin{array}{c}\Psi_{R} \\ \Psi_{L}\end{array}\right), \bar{\Psi}=\Psi^{\dagger} \gamma^{\mathrm{y}}$. We choose the Dirac matrices as

$$
\gamma^{\mathrm{x}}=\left(\begin{array}{cc}
0 & i \\
-i & 0
\end{array}\right), \gamma^{\mathrm{y}}=\left(\begin{array}{ll}
0 & 1 \\
1 & 0
\end{array}\right)
$$

The two-point function that we are interested in can be represented by the appropriately regularized Euclidean functional integral

$$
\left\langle\mathcal{O}_{\alpha}(x) \mathcal{O}_{\alpha^{\prime}}(y)\right\rangle=\int_{\mathcal{F}_{\alpha, \alpha^{\prime}}}[\mathcal{D} \Psi \mathcal{D} \bar{\Psi}] e^{-\mathcal{A}} .
$$

The integration is over the space $\mathcal{F}_{\alpha, \alpha^{\prime}}$ of field configurations vanishing on the boundary of the disk and such that $\Psi, \bar{\Psi}$ acquire phases when continued counterclockwise around the points $x$ and $y$ :

$$
\mathcal{F}_{\alpha, \alpha^{\prime}}:\left\{\begin{array}{l}
\text { around } x: \Psi \rightarrow e^{2 \pi i \alpha} \Psi, \bar{\Psi} \rightarrow e^{-2 \pi i \alpha} \bar{\Psi} \\
\text { around } y: \Psi \rightarrow e^{2 \pi i \alpha^{\prime}} \Psi, \bar{\Psi} \rightarrow e^{-2 \pi i \alpha^{\prime}} \bar{\Psi}
\end{array}\right.
$$




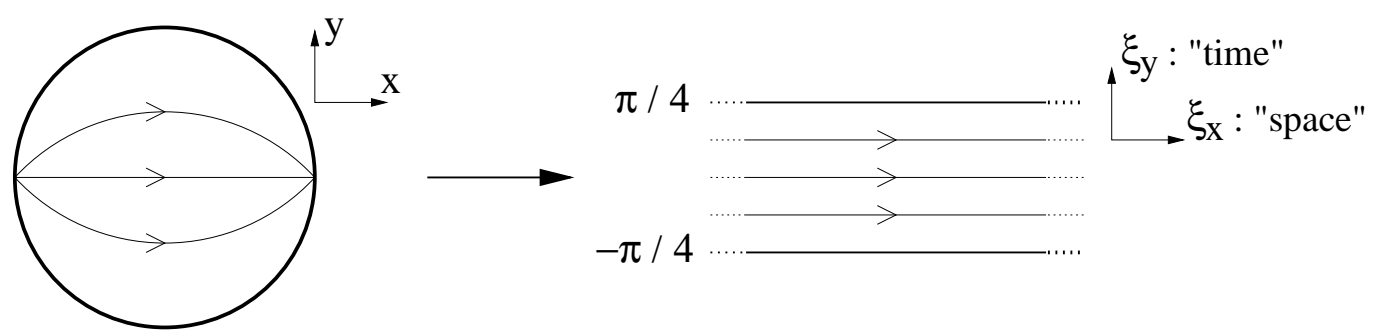

Figure 1: mapping from the Poincaré disk to the strip. Lines with an arrow represent orbits of $\mathbf{K}$.

\section{Hilbert space}

An expansion of the functional integral (2.3) for large geodesic distance between the points $x$ and $y$ is most conveniently obtained by using the operator formalism. We will construct a Hilbert space $\mathcal{H}$ on which this functional integral is represented by a vacuum expectation value $\left\langle v a c\left|\mathcal{T}\left[\mathcal{O}_{\alpha}(x) \mathcal{O}_{\alpha^{\prime}}(y)\right]\right| v a c\right\rangle$, where $|v a c\rangle$ is a $S U(1,1)$ invariant vacuum implementing vanishing boundary conditions and where the fields $\mathcal{O}_{\alpha}(x)$ and $\mathcal{O}_{\alpha^{\prime}}(y)$ act on $\mathcal{H}$ as operators implementing the quasi-periodicity conditions (2.4). The symbol $\mathcal{T}$ here denotes an appropriate "time"-ordering, described below. The large distance expansion will be obtained by inserting between the fields $\mathcal{O}_{\alpha}(x)$ and $\mathcal{O}_{\alpha^{\prime}}(y)$ a resolution of the identity in terms of a basis of states that diagonalize a non-compact subgroup of the $S U(1,1)$ isometry group of the Poincaré disk.

We define the Hilbert space $\mathcal{H}$ by quantizing the theory on curves that are orbits of the non-compact subgroup K:

$$
\mathbf{K}: g_{q}=\left(\begin{array}{cc}
\cosh (q) & \sinh (q) \\
\sinh (q) & \cosh (q)
\end{array}\right), q \in \mathbb{R} .
$$

Translations in the "time" direction, perpendicular to these curves, are not isometries, so that the Hamiltonian is not stationary. Translations in the "space" direction, along these curves, are isometries, and a basis for $\mathcal{H}$ will be obtained by diagonalizing the generator of such translations. In order to construct $\mathcal{H}$, we first map the Poincaré disk onto the strip (see Figure 1):

$$
z=\tanh (\xi), \bar{z}=\tanh (\bar{\xi})
$$

with

$$
\xi=\xi_{\mathrm{x}}+i \xi_{\mathrm{y}}, \xi_{\mathrm{x}} \in \mathbb{R},-\pi / 4<\xi_{\mathrm{y}}<\pi / 4 .
$$

The action (2.2) for the fermion fields $\Psi_{s}=\left(\begin{array}{c}\left(\Psi_{s}\right)_{R} \\ \left(\Psi_{s}\right)_{L}\end{array}\right)$ on the strip is

$$
\mathcal{A}=\int d \xi_{\mathrm{x}} d \xi_{\mathrm{y}} \bar{\Psi}_{s}\left(\gamma^{\mathrm{x}} \frac{\partial}{\partial \xi_{\mathrm{x}}}+\gamma^{\mathrm{y}} \frac{\partial}{\partial \xi_{\mathrm{y}}}+\frac{2 \mu}{\cos \left(2 \xi_{\mathrm{y}}\right)}\right) \Psi_{s}
$$

where $\bar{\Psi}_{s}=\Psi_{s}^{\dagger} \gamma^{\mathrm{y}}$. Elements $g_{q}$ of the subgroup $\mathbf{K}$ are translations parallel to the strip: $\xi_{\mathrm{x}} \rightarrow \xi_{\mathrm{x}}+q$, so that the quantization space is specified by the curves $\xi_{\mathrm{y}}=$ const. The coordinate $\xi_{\mathrm{y}}$ is the Euclidean "time". Correlation functions of local operators are expressed as vacuum expectation values of "time"ordered products, for instance

$$
\left\langle\mathcal{O}_{\alpha_{1}}\left(\xi_{\mathrm{x}_{1}}, \xi_{\mathrm{y}_{1}}\right) \mathcal{O}_{\alpha_{2}}\left(\xi_{\mathrm{x}_{2}}, \xi_{\mathrm{y}_{2}}\right) \cdots\right\rangle=\left\langle v a c\left|\mathcal{T}\left[\mathcal{O}_{\alpha_{1}}\left(\xi_{\mathrm{x}_{1}}, \xi_{\mathrm{y}_{1}}\right) \mathcal{O}_{\alpha_{2}}\left(\xi_{\mathrm{x}_{2}}, \xi_{\mathrm{y}_{2}}\right) \cdots\right]\right| v a c\right\rangle,
$$

where the "time"-ordering $\mathcal{T}$ means that operators have to be ordered form left to right in decreasing values of their variable $\xi_{\mathrm{y}}$. The Hilbert space $\mathcal{H}$ is a module for the canonical equal- "time" anti-commutation relations

$$
\left\{\Psi_{s}\left(\xi_{\mathrm{x}}, \xi_{\mathrm{y}}\right), \Psi_{s}^{\dagger}\left(\xi_{\mathrm{x}}^{\prime}, \xi_{\mathrm{y}}\right)\right\}=\mathbf{1} \delta\left(\xi_{\mathrm{x}}-\xi_{\mathrm{x}}^{\prime}\right),
$$


where now $\Psi_{s}\left(\xi_{\mathrm{x}}, \xi_{\mathrm{y}}\right), \Psi_{s}^{\dagger}\left(\xi_{\mathrm{x}}, \xi_{\mathrm{y}}\right)$ are fermion operators acting on $\mathcal{H}$, related on different "time" slices $\xi_{\mathrm{y}}=$ const by the equations of motion. The vacuum state $|v a c\rangle \in \mathcal{H}$ is $S U(1,1)$ invariant, and satisfies the conditions that the fermion operators vanish on it at early "times" $\xi_{\mathrm{y}} \rightarrow-\pi / 4$ and on its dual $\langle v a c|$ at late "times" $\xi_{\mathrm{y}} \rightarrow \pi / 4$ :

$$
\begin{aligned}
\lim _{\xi_{\mathrm{y}} \rightarrow-\pi / 4} \Psi_{s}\left(\xi_{\mathrm{x}}, \xi_{\mathrm{y}}\right)|v a c\rangle & =\lim _{\xi_{\mathrm{y}} \rightarrow-\pi / 4} \Psi_{s}^{\dagger}\left(\xi_{\mathrm{x}}, \xi_{\mathrm{y}}\right)|v a c\rangle=0 \\
\lim _{\xi_{\mathrm{y}} \rightarrow \pi / 4}\langle v a c| \Psi_{s}\left(\xi_{\mathrm{x}}, \xi_{\mathrm{y}}\right) & =\lim _{\xi_{\mathrm{y}} \rightarrow \pi / 4}\langle v a c| \Psi_{s}^{\dagger}\left(\xi_{\mathrm{x}}, \xi_{\mathrm{y}}\right)=0 .
\end{aligned}
$$

A basis for the Hilbert space $\mathcal{H}$ diagonalizing the subgroup $\mathbf{K}$ is obtained by considering a module for appropriate modes $A_{\epsilon}(\omega), A_{\epsilon}^{\dagger}(\omega)$ of the Fermion operators $\Psi_{s}\left(\xi_{\mathrm{x}}, \xi_{\mathrm{y}}\right), \Psi_{s}^{\dagger}\left(\xi_{\mathrm{x}}, \xi_{\mathrm{y}}\right)$, where $\epsilon= \pm$ represents the $U(1)$ charge. These modes appear in an expansion in terms of partial waves satisfying the equations of motion and diagonalizing the spin- $\frac{1}{2}$ action of the subgroup $\mathbf{K}$ :

$$
\begin{aligned}
& \Psi_{s}\left(\xi_{\mathrm{x}}, \xi_{\mathrm{y}}\right)=\int d \omega \rho(\omega)\left(A_{-}^{\dagger}(\omega) e^{i \omega \xi_{\mathrm{x}}} \bar{P}_{-}\left(\omega, \xi_{\mathrm{y}}\right)+A_{+}(\omega) e^{-i \omega \xi_{\mathrm{x}}} P_{+}\left(\omega, \xi_{\mathrm{y}}\right)\right) \\
& \Psi_{s}^{\dagger}\left(\xi_{\mathrm{x}}, \xi_{\mathrm{y}}\right)=\int d \omega \rho(\omega)\left(A_{+}^{\dagger}(\omega) e^{i \omega \xi_{\mathrm{x}}} \bar{P}_{+}\left(\omega, \xi_{\mathrm{y}}\right)+A_{-}(\omega) e^{-i \omega \xi_{\mathrm{x}}} P_{-}\left(\omega, \xi_{\mathrm{y}}\right)\right),
\end{aligned}
$$

where the integrations are from $-\infty$ to $\infty$. We choose the measure

$$
\rho(\omega)=\frac{\Gamma\left(\frac{1}{2}+\mu+i \frac{\omega}{2}\right) \Gamma\left(\frac{1}{2}+\mu-i \frac{\omega}{2}\right)}{2 \pi \Gamma\left(\frac{1}{2}+\mu\right)^{2}}
$$

in order for the partial waves to be entire functions of the spectral parameter $\omega$; this analytical property will be used later. The solutions for the partial waves can be written:

$$
P_{+}\left(\omega, \xi_{\mathrm{y}}\right)=\left(\begin{array}{c}
2^{-2 \mu-\frac{1}{2}} e^{-i \frac{\pi}{2}\left(\mu+\frac{1}{2}-i \frac{\omega}{2}\right)}\left(1+e^{4 i \xi_{\mathrm{y}}}\right)^{\mu} e^{\omega \xi_{\mathrm{y}}} F\left(\mu, \frac{1}{2}+\mu-i \frac{\omega}{2} ; 1+2 \mu ; 1+e^{4 i \xi_{\mathrm{y}}}\right) \\
2^{-2 \mu-\frac{1}{2}} e^{-i \frac{\pi}{2}\left(\mu+\frac{1}{2}+i \frac{\omega}{2}\right)}\left(1+e^{4 i \xi_{\mathrm{y}}}\right)^{\mu} e^{-\omega \xi_{\mathrm{y}}} F\left(\mu, \frac{1}{2}+\mu+i \frac{\omega}{2} ; 1+2 \mu ; 1+e^{4 i \xi_{\mathrm{y}}}\right)
\end{array}\right)
$$

and

$$
\bar{P}_{\epsilon}\left(\omega, \xi_{\mathrm{y}}\right)=\left(P_{\epsilon}\left(\omega,-\xi_{\mathrm{y}}\right)\right)^{\dagger},\left(P_{-}\left(\omega, \xi_{\mathrm{y}}\right)\right)^{t}=\left(\begin{array}{cc}
1 & 0 \\
0 & -1
\end{array}\right) P_{+}\left(\omega, \xi_{\mathrm{y}}\right)
$$

where ${ }^{t}$ means transpose, and where $F(a, b ; c ; z)$ is Gauss's hypergeometric function on a Riemann sheet delimited by the branch cut $(-\infty, 1]$, with $\lim _{z \rightarrow 0, \Im m(z)>0} F(a, b ; c ; z)=1$. With this choice of solutions, the partial waves $P_{ \pm}\left(\omega, \xi_{\mathrm{y}}\right)$ vanish on the upper boundary $\xi_{\mathrm{y}}=\pi / 4$ of the strip, and $\bar{P}_{ \pm}\left(\omega, \xi_{\mathrm{y}}\right)$ vanish on the lower boundary $\xi_{\mathrm{y}}=-\pi / 4$ of the strip.

The canonical anti-commutation relations for the fermion operators imply the following anti-commutation relations for the modes:

$$
\left\{A_{\epsilon}(\omega), A_{\epsilon^{\prime}}^{\dagger}\left(\omega^{\prime}\right)\right\}=\frac{1}{\rho(\omega)} \delta\left(\omega-\omega^{\prime}\right) \delta_{\epsilon, \epsilon^{\prime}},\left\{A_{\epsilon}(\omega), A_{\epsilon^{\prime}}\left(\omega^{\prime}\right)\right\}=\left\{A_{\epsilon}^{\dagger}(\omega), A_{\epsilon^{\prime}}^{\dagger}\left(\omega^{\prime}\right)\right\}=0 .
$$

The Hilbert space is the Fock space over this algebra, and the asymptotic conditions (3.2) specify the vacuum $|v a c\rangle$ :

$$
A_{ \pm}(\omega)|v a c\rangle=0
$$

which we normalize to $\langle v a c \mid v a c\rangle=1$. A complete basis is given by

$$
\left|\omega_{1}, \ldots, \omega_{n}\right\rangle_{\epsilon_{1}, \ldots, \epsilon_{n}}=A_{\epsilon_{1}}^{\dagger}\left(\omega_{1}\right) \cdots A_{\epsilon_{n}}^{\dagger}\left(\omega_{n}\right)|v a c\rangle
$$

for a given ordering of the $\omega_{j}$ 's, for instance the "in-ordering" $\omega_{1}<\cdots<\omega_{n}$.

The states constructed diagonalize the subgroup $\mathbf{K}$ :

$$
\hat{g}_{q}\left|\omega_{1}, \ldots, \omega_{n}\right\rangle_{\epsilon_{1}, \ldots, \epsilon_{n}}=e^{-i q\left(\omega_{1}+\cdots+\omega_{n}\right)}\left|\omega_{1}, \ldots, \omega_{n}\right\rangle_{\epsilon_{1}, \ldots, \epsilon_{n}}
$$


as well as the $U(1)$ charge, with eigenvalue $\epsilon_{1}+\cdots+\epsilon_{n}$. Here and below we use the notation $\hat{g}$ for representing the action of the group element $g$ on the space $\mathcal{H}$. These states can be used to obtain a resolution of the identity on $\mathcal{H}$ :

$$
\mathbf{1}_{\mathcal{H}}=\sum_{n=0}^{\infty} \frac{1}{n !} \sum_{\epsilon_{1}, \ldots, \epsilon_{n}} \int\left(\prod_{j=1}^{n} d \omega_{j} \rho\left(\omega_{j}\right)\right)\left|\omega_{1}, \ldots, \omega_{n}\right\rangle_{\epsilon_{1}, \ldots, \epsilon_{n}} \quad \epsilon_{n}, \ldots, \epsilon_{1}\left\langle\omega_{n}, \ldots, \omega_{1}\right|,
$$

where states with different orderings of $\omega_{j}$ 's than the in-ordering $\omega_{1}<\cdots<\omega_{n}$ are given by the same expression (3.8) in terms of modes. They differ from states with in-ordering by a sign through the anti-commutation relations (3.7).

On the Hilbert space $\mathcal{H}$, the scaling fields $\mathcal{O}_{\alpha}(x)$ act as appropriately regularized exponentials of line integrals of the $U(1)$ current:

$$
\mathcal{O}_{\alpha}(x) \mapsto \pi_{\mathcal{H}}\left(\exp \left[2 \pi i \alpha \int_{\mathcal{C}_{x}} d x^{\mu} \epsilon_{\mu, \nu} \bar{\Psi} \gamma^{\nu} \Psi\right]\right)
$$

where $\mathcal{C}_{x}$ is a path from the position $x$ to the boundary of the disk and $\pi_{\mathcal{H}}$ is the representation map on the space $\mathcal{H}$ (for a precise definition of the action of similar scaling fields on the standard Hilbert space of a Dirac theory on flat background, see for instance [7]). The resolution of the identity on $\mathcal{H}$ then gives the long geodesic distance expansion of the two-point function $\left\langle\mathcal{O}_{\alpha}(x) \mathcal{O}_{\alpha^{\prime}}(y)\right\rangle=\left\langle\operatorname{vac}\left|\mathcal{T}\left[\mathcal{O}_{\alpha}(x) \mathcal{O}_{\alpha^{\prime}}(y)\right]\right| v a c\right\rangle$. Using the fact that the one-point function $\left\langle\mathcal{O}_{\alpha}\right\rangle=\left\langle v a c\left|\mathcal{O}_{\alpha}\right| v a c\right\rangle$ is non-zero and defining the function

$$
F_{\alpha}\left(\omega_{1}, \ldots, \omega_{n}\right)_{\epsilon_{1}, \ldots, \epsilon_{n}} \equiv \frac{\left\langle v a c\left|\mathcal{O}_{\alpha}(0)\right| \omega_{1}, \ldots, \omega_{n}\right\rangle_{\epsilon_{1}, \ldots, \epsilon_{n}}}{\left\langle v a c\left|\mathcal{O}_{\alpha}\right| v a c\right\rangle}
$$

we have

$$
\begin{aligned}
\left\langle\mathcal{O}_{\alpha}(x) \mathcal{O}_{\alpha^{\prime}}(y)\right\rangle= & \left\langle\mathcal{O}_{\alpha}\right\rangle\left\langle\mathcal{O}_{\alpha^{\prime}}\right\rangle \sum_{n=0}^{\infty} \frac{1}{n !} \sum_{\epsilon_{1}, \ldots, \epsilon_{n}} \int\left(\prod_{j=1}^{n} d \omega_{j} \rho\left(\omega_{j}\right)\right) \times \\
& \times F_{\alpha}\left(\omega_{1}, \ldots, \omega_{n}\right)_{\epsilon_{1}, \ldots, \epsilon_{n}}\left(F_{-\alpha^{\prime}}\left(\omega_{1}, \ldots, \omega_{n}\right)_{\epsilon_{1}, \ldots, \epsilon_{n}}\right)^{*} e^{-i\left(\omega_{1}+\cdots+\omega_{n}\right) \frac{\mathrm{d}(x, y)}{2 R}}
\end{aligned}
$$

In order to obtain this formula, one first brings $y$ to the origin and $x$ to the real axis inside the Poincaré disk; this can always be done by $S U(1,1)$ invariance of the correlator. Since the subgroup $\mathbf{K}$ generates geodesic translations along the real axis inside the Poincaré disk, matrix elements of the operator $\mathcal{O}_{\alpha}(x)$ are related to those of $\mathcal{O}_{\alpha}(0)$ by an exponential factor involving the geodesic distance $\mathrm{d}(x, 0)$. Using $S U(1,1)$ invariance again, one can replace this by $\mathrm{d}(x, y)$ for the correlator of fields at arbitrary points $x$ and $y$. In the next section we calculate the matrix elements $\left\langle v a c\left|\mathcal{O}_{\alpha}(0)\right| \omega_{1}, \ldots, \omega_{n}\right\rangle_{\epsilon_{1}, \ldots, \epsilon_{n}}$, which we call "form factors" of the scaling fields $\mathcal{O}_{\alpha}(0)$.

\section{Angular quantization and form factors}

In order to construct form factors of local fields in quantum integrable models, in [13 14 the authors used the idea of embedding the Hilbert space $\mathcal{H}$ of a quantum field theory in two-dimensional flat space-time into a tensor product

$$
\mathcal{H}_{A} \otimes \mathcal{H}_{A}^{*}
$$

of the Hilbert space of angular quantization $\mathcal{H}_{A}$ and its dual $\mathcal{H}_{A}^{*}$. In angular quantization, the Hamiltonian is taken as the generator of rotations around a given point. Form factors of local fields can then be constructed as traces on the angular Hilbert space $\mathcal{H}_{A}$. We will make a similar construction in order to obtain the matrix elements $\left\langle\operatorname{vac}\left|\mathcal{O}_{\alpha}(0)\right| \omega_{1}, \ldots, \omega_{n}\right\rangle_{\epsilon_{1}, \ldots, \epsilon_{n}}$. The angular Hamiltonian will be taken as the generator of rotations around the center of the Poincaré disk; it is the generator for the compact subgroup of the $S U(1,1)$ isometry group of the Poincaré disk. 
We first briefly develop the formalism of angular quantization [12, 14, 15, for our theory. Angular quantization is done in conformal polar coordinates $(\eta, \theta)$, where $\theta$ is the Euclidean "time" and $-\infty<$ $\eta<0$ :

$$
z=e^{\eta+i \theta}, \bar{z}=e^{\eta-i \theta}
$$

The fermion fields $\Psi_{\text {pol }}=\left(\begin{array}{c}\left(\Psi_{\text {pol }}\right)_{R} \\ \left(\Psi_{\text {pol }}\right)_{L}\end{array}\right)$ in these coordinates enter the Dirac action as

$$
\mathcal{A}=\int_{0}^{2 \pi} d \theta \int_{-\infty}^{0} d \eta \bar{\Psi}_{p o l}\left(\gamma^{\eta} \partial_{\eta}+\gamma^{\theta} \partial_{\theta}-\frac{\mu}{\sinh (\eta)}\right) \Psi_{p o l}
$$

where $\bar{\Psi}_{p o l}=\Psi_{\text {pol }}^{\dagger} \gamma^{\theta}$ and $\gamma^{\eta}, \gamma^{\theta}$ are, respectively, the same matrices as $\gamma^{\mathrm{x}}, \gamma^{\mathrm{y}}$. The angular Hamiltonian derived from this action is

$$
H_{A}=\int_{-\infty}^{0} d \eta: \Psi_{p o l}^{\dagger} \gamma^{\theta}\left(\gamma^{\eta} \partial_{\eta}-\frac{\mu}{\sinh (\eta)}\right) \Psi_{p o l}:
$$

where $\Psi_{\text {pol }}(\eta)$ and $\Psi_{\text {pol }}^{\dagger}(\eta)$ are now operators on the angular Hilbert space $\mathcal{H}_{A}$ satisfying the canonical anti-commutation relation

$$
\left\{\Psi_{\text {pol }}(\eta), \Psi_{\text {pol }}^{\dagger}\left(\eta^{\prime}\right)\right\}=\mathbf{1} \delta\left(\eta-\eta^{\prime}\right) .
$$

The $\eta$-dependent mass term in the Hamiltonian (4.3) produces a "mass barrier" effect somewhat similar to the effect of the mass term in the theory on flat space [12]; it prevents the fermions from approaching too much the boundary of the disk. More precisely, it imposes vanishing asymptotic conditions for the fermion fields when $\mu>\frac{1}{2}$ (such asymptotic conditions are in fact allowed for all $\mu>0$ ). With these asymptotic conditions, the Hamiltonian is diagonalized by the decomposition

$$
\Psi_{p o l}(\eta, \theta)=\int_{-\infty}^{\infty} \frac{d \nu}{\sqrt{2 \pi}} c_{\nu} \mathcal{U}_{\nu}(\eta) e^{-\nu \theta}, \Psi_{p o l}^{\dagger}(\eta, \theta)=\int_{-\infty}^{\infty} \frac{d \nu}{\sqrt{2 \pi}} c_{\nu}^{\dagger} \mathcal{U}_{\nu}^{\dagger}(\eta) e^{\nu \theta}
$$

in terms of partial waves

$$
\mathcal{U}_{\nu}=\left(\begin{array}{c}
u_{\nu} \\
v_{\nu}
\end{array}\right)
$$

with

$$
\begin{aligned}
& u_{\nu}=\frac{\Gamma(1+\mu) \Gamma\left(\frac{1}{2}+\mu-i \nu\right)}{\Gamma(1+2 \mu) \Gamma\left(\frac{1}{2}-i \nu\right)} e^{i \nu \eta}\left(1-e^{2 \eta}\right)^{\mu} F\left(\mu, \frac{1}{2}+\mu+i \nu ; 1+2 \mu ; 1-e^{2 \eta}\right) \\
& v_{\nu}=-i \frac{\Gamma(1+\mu) \Gamma\left(\frac{1}{2}+\mu-i \nu\right)}{\Gamma(1+2 \mu) \Gamma\left(\frac{1}{2}-i \nu\right)} e^{-i \nu \eta}\left(1-e^{2 \eta}\right)^{\mu} F\left(\mu, \frac{1}{2}+\mu-i \nu ; 1+2 \mu ; 1-e^{2 \eta}\right),
\end{aligned}
$$

where $F(a, b ; c ; z)$ is Gauss's hypergeometric function on its principal branch. In (4.4), the operators $c_{\nu}, c_{\nu}^{\dagger}$ satisfy the canonical anti-commutation relations

$$
\left\{c_{\nu}^{\dagger}, c_{\nu^{\prime}}\right\}=\delta\left(\nu-\nu^{\prime}\right) .
$$

The angular Hilbert space $\mathcal{H}_{A}$ is the fermionic Fock space over this algebra, with vacuum vector $|0\rangle_{A}$ defined by

$$
c_{\nu}|0\rangle_{A}=0 \quad(\nu>0), \quad c_{\nu}^{\dagger}|0\rangle_{A}=0 \quad(\nu<0) .
$$

With an appropriate normal-ordering, the Hamiltonian takes the form

$$
H_{A}=\int_{0}^{\infty} d \nu \nu\left(c_{\nu}^{\dagger} c_{\nu}+c_{-\nu} c_{-\nu}^{\dagger}\right)
$$

We now consider the embedding $\mathcal{H} \hookrightarrow \mathcal{H}_{A} \otimes \mathcal{H}_{A}^{*}$ that will allow us to calculate form factors of scaling fields $\left\langle\text { vac }\left|\mathcal{O}_{\alpha}(0)\right| \omega_{1}, \ldots, \omega_{n}\right\rangle_{\epsilon_{1}, \ldots, \epsilon_{n}}$. The embedding is described by identifying vectors in the Hilbert space $\mathcal{H}$ with endomorphisms on the angular Hilbert space $\mathcal{H}_{A}$ :

$$
\left|\omega_{1}, \ldots, \omega_{n}\right\rangle_{\epsilon_{1}, \ldots, \epsilon_{n}} \equiv a_{\epsilon_{1}}\left(\omega_{1}\right) \cdots a_{\epsilon_{n}}\left(\omega_{n}\right) e^{-\pi H_{A}},
$$


where the operators $a_{\epsilon}(\omega) \in \operatorname{End}\left(\mathcal{H}_{A}\right)$ are to be determined. Notice that the vacuum $|v a c\rangle$ is identified with $e^{-\pi H_{A}}$. The scalar product on $\mathcal{H}$ is identified with the canonical scalar product on the space $\operatorname{End}\left(\mathcal{H}_{A}\right)$, which coincides with the expression of correlation functions as traces on $\mathcal{H}_{A}$ :

$$
\langle u \mid v\rangle \equiv \frac{\operatorname{Tr}\left(U^{\dagger} V\right)}{\operatorname{Tr}\left(e^{-2 \pi H_{A}}\right)} \text { if }|u\rangle \equiv U,|v\rangle \equiv V .
$$

The representation of a field on $\mathcal{H}$ is identified with its representation on $\mathcal{H}_{A}$ :

$$
\pi_{\mathcal{H}}(\mathcal{O})|u\rangle \equiv \pi_{A}(\mathcal{O}) U \text { if }|u\rangle \equiv U
$$

where $\pi_{A}$ is the representation map on the space $\mathcal{H}_{A}$.

The operators $a_{\epsilon}(\omega)$ can be fixed by imposing two conditions. First, the operators on $\mathcal{H}_{A}$ representing fields at the center of the disk that are mutually local with the fermion field must commute (if they are bosonic) or anti-commute (if they are fermionic) with the operators $a_{\epsilon}(\omega)$. It is sufficient to impose this condition with the fermion operators themselves:

$$
\left\{a_{\epsilon}(\omega), \Psi_{\text {pol }}(\eta \rightarrow-\infty)\right\}=\left\{a_{\epsilon}(\omega), \Psi_{\text {pol }}^{\dagger}(\eta \rightarrow-\infty)\right\}=0 .
$$

Second, the embedding (4.7) must reproduce the form factors of fermion fields $\left\langle v a c\left|\Psi_{s}\left(\xi_{\mathrm{x}}, \xi_{\mathrm{y}}\right)\right| \omega\right\rangle_{+}=$ $e^{-i \omega \xi_{\mathrm{x}}} P_{+}\left(\omega, \xi_{\mathrm{y}}\right)$ and $\left\langle v a c\left|\Psi_{s}^{\dagger}\left(\xi_{\mathrm{x}}, \xi_{\mathrm{y}}\right)\right| \omega\right\rangle_{-}=e^{-i \omega \xi_{\mathrm{x}}} P_{-}\left(\omega, \xi_{\mathrm{y}}\right)$ obtained from the partial wave decomposition (3.3). As shown in Appendix A, these two conditions are satisfied by the operators

$$
\begin{gathered}
a_{+}(\omega)=\int_{-\infty}^{\infty} d \nu g(\nu ; \omega) c_{\nu}^{\dagger}, \quad a_{-}(\omega)=\int_{-\infty}^{\infty} d \nu g(\nu ; \omega) c_{-\nu} \\
g(\nu ; \omega)=\sqrt{\pi} 2^{-\mu} e^{i \frac{\pi}{2}\left(\mu+\frac{1}{2}-i \frac{\omega}{2}\right)} \frac{e^{-\pi \nu} \Gamma\left(\frac{1}{2}+\mu+i \nu\right)}{\Gamma(1+\mu) \Gamma\left(\frac{1}{2}+i \nu\right)} F\left(\mu+\frac{1}{2}+i \nu, \mu+\frac{1}{2}-i \frac{\omega}{2} ; 1+2 \mu ; 2-i 0\right) .
\end{gathered}
$$

Here and below we use the notation

$$
F(a, b ; c ; 2 \pm i 0)=\lim _{\varepsilon \rightarrow 0^{+}} F(a, b ; c ; 2 \pm i \varepsilon),
$$

where on the right hand side $F(a, b ; c ; z)$ is Gauss's hypergeometric function on its principal branch. We believe that the solution (4.10) to the two conditions above is unique. One can verify for instance that

$$
\frac{\operatorname{Tr}\left(e^{-2 \pi H_{A}} \pi_{A}\left(\Psi_{s}\left(\xi_{\mathrm{x}}, \xi_{\mathrm{y}}\right)\right) a_{+}(\omega)\right)}{\operatorname{Tr}\left(e^{-2 \pi H_{A}}\right)}=e^{-i \omega \xi_{\mathrm{x}}} P_{+}\left(\omega, \xi_{\mathrm{y}}\right)
$$

such expression can be calculated by using the cyclic properties of the traces and the anti-commutation relations for the modes $c_{\nu}, c_{\nu}^{\dagger}$. As is shown in Appendix B.1, the operators $a_{\epsilon}(\omega)$ anti-commute among themselves:

$$
\left\{a_{\epsilon_{1}}\left(\omega_{1}\right), a_{\epsilon_{2}}\left(\omega_{2}\right)\right\}=0 .
$$

Also, since they are linear combinations of free modes, traces of products of such operators satisfy Wick's theorem. In particular we have the following contractions:

$$
\begin{aligned}
& \frac{\operatorname{Tr}\left(e^{-2 \pi H_{A}} a_{\epsilon}^{\dagger}(\omega) a_{\epsilon^{\prime}}\left(\omega^{\prime}\right)\right)}{\operatorname{Tr}\left(e^{-2 \pi H_{A}}\right)}=\frac{1}{\rho(\omega)} \delta\left(\omega-\omega^{\prime}\right) \delta_{\epsilon, \epsilon^{\prime}} \\
& \frac{\operatorname{Tr}\left(e^{-2 \pi H_{A}} a_{\epsilon}(\omega) a_{\epsilon^{\prime}}\left(\omega^{\prime}\right)\right)}{\operatorname{Tr}\left(e^{-2 \pi H_{A}}\right)}=0
\end{aligned}
$$

Since these contractions are respectively $\epsilon_{\epsilon}\left\langle\omega \mid \omega^{\prime}\right\rangle_{\epsilon^{\prime}}$ and $\left\langle v a c \mid \omega, \omega^{\prime}\right\rangle_{\epsilon, \epsilon^{\prime}}$ and since any form factor of fermion fields in $\mathcal{H}$ can be evaluated by Wick's theorem, the embedding (4.7) reproduces all form factors of fermion fields. 
From the expression (4.10) one can verify the following identity:

$$
a_{\epsilon}^{\dagger}(\omega)=e^{\pi H_{A}} a_{-\epsilon}(\omega) e^{-\pi H_{A}} .
$$

This is the analogue of crossing symmetry present in theories on flat space. For the scaling fields $\mathcal{O}_{\alpha}$, which are scalar and have the property $\mathcal{O}_{\alpha}^{\dagger}=\mathcal{O}_{-\alpha}$, this leads for instance to

$$
\left(F_{\alpha}\left(\omega_{1}, \ldots, \omega_{n}\right)_{\epsilon_{1}, \ldots, \epsilon_{n}}\right)^{*}=F_{-\alpha}\left(\omega_{n}, \ldots, \omega_{1}\right)_{-\epsilon_{n}, \ldots,-\epsilon_{1}}
$$

using the notation (3.10).

From the representation (3.9) of the scaling fields on $\mathcal{H}$, we have the following embedding:

$$
\mathcal{O}_{\alpha}(0)|u\rangle \equiv e^{2 \pi i \alpha Q} U \text { if }|u\rangle \equiv U,
$$

where $Q$ is the $U(1)$ charge in angular quantization:

$$
Q=\int_{0}^{\infty} d \nu\left(c_{\nu}^{\dagger} c_{\nu}-c_{-\nu} c_{-\nu}^{\dagger}\right)
$$

Using this embedding, form factors of the scaling fields $\mathcal{O}_{\alpha}$ are given heuristically by the following traces:

$$
\left\langle v a c\left|\mathcal{O}_{\alpha}(0)\right| \omega_{1}, \ldots, \omega_{n}\right\rangle_{\epsilon_{1}, \ldots, \epsilon_{n}}=\frac{\operatorname{Tr}\left(e^{-2 \pi H_{A}+2 \pi i \alpha Q} a_{\epsilon_{1}}\left(\omega_{1}\right) \cdots a_{\epsilon_{n}}\left(\omega_{n}\right)\right)}{\operatorname{Tr}\left(e^{-2 \pi H_{A}}\right)} .
$$

Both traces in the ratio above are ill-defined and need some regularization $\operatorname{Tr} \rightarrow \operatorname{Tr}{ }_{\varepsilon}$. In the next section we will regularize such traces by doing the angular quantization on a Poincaré disk from which a small disk of radius $\varepsilon$ around the origin has been removed. As the regularization parameter disappears $\varepsilon \rightarrow 0$, the resulting ratio of traces above is then singular and goes as $\varepsilon^{\alpha^{2}}$. We can cancel out this singularity by considering normalized form factors:

$$
\frac{\left\langle v a c\left|\mathcal{O}_{\alpha}(0)\right| \omega_{1}, \ldots, \omega_{n}\right\rangle_{\epsilon_{1}, \ldots, \epsilon_{n}}}{\left\langle v a c\left|\mathcal{O}_{\alpha}\right| v a c\right\rangle}=\frac{\operatorname{Tr}\left(e^{-2 \pi H_{A}+2 \pi i \alpha Q} a_{\epsilon_{1}}\left(\omega_{1}\right) \cdots a_{\epsilon_{n}}\left(\omega_{n}\right)\right)}{\operatorname{Tr}\left(e^{-2 \pi H_{A}+2 \pi i \alpha Q}\right)} .
$$

These can be calculated without explicit reference to a regularization procedure, simply by using, as above, the cyclic properties of the trace and the anti-commutation relations for the modes $c_{\nu}, c_{\nu}^{\dagger}$. The calculation of the one-point function requires the use of an explicit regularization procedure:

$$
\left\langle\mathcal{O}_{\alpha}\right\rangle=\left\langle v a c\left|\mathcal{O}_{\alpha}\right| v a c\right\rangle=\lim _{\varepsilon \rightarrow 0} \varepsilon^{-\alpha^{2}} \frac{\operatorname{Tr}_{\varepsilon}\left(e^{-2 \pi H_{A}+2 \pi i \alpha Q}\right)}{\operatorname{Tr}_{\varepsilon}\left(e^{-2 \pi H_{A}}\right)},
$$

and will be done in the next section.

The traces 4.15) are calculated in Appendix B. In particular, the "two-particle" form factors are given in (B.4). Other "multi-particle" form factors of scaling fields $\mathcal{O}_{\alpha}(0)$ can be constructed from these "twoparticle" form factors by Wick's theorem, as in (B.9). In Appendix C, we verify that the expression (B.4) for the "two-particle" form factors specializes to the known expression for form factors in the flat-space limit.

\section{One-point function}

We now calculate the one-point function $\left\langle O_{\alpha}\right\rangle$. This is a simple generalization of the calculation done in [11, 12] for similar vacuum expectation values in flat space.

We regularize the traces $\operatorname{Tr} \rightarrow \operatorname{Tr}_{\varepsilon}$ by cutting a small disk of radius $\varepsilon$ around the origin and considering the angular quantization of the theory (2.2) on the resulting annulus with the boundary conditions

$$
\left[\left(\Psi_{p o l}\right)_{R}(\eta)-\left(\Psi_{p o l}\right)_{L}(\eta)\right]_{\eta=\ln \tanh \left(\frac{\varepsilon}{2 R}\right)}=\left[\left(\Psi_{p o l}\right)_{R}^{\dagger}(\eta)-\left(\Psi_{p o l}\right)_{L}^{\dagger}(\eta)\right]_{\eta=\ln \tanh \left(\frac{\varepsilon}{2 R}\right)}=0 .
$$


Then the one-point function $\left\langle\mathcal{O}_{\alpha}\right\rangle$ can be expressed as (4.16), where the $U(1)$ charge $Q$ in the regularized theory is

$$
Q=\int_{\ln \tanh \left(\frac{\varepsilon}{2 R}\right)}^{0} d \eta: \Psi_{p o l}^{\dagger} \Psi_{p o l}:
$$

which specializes to (4.14) in the limit $\varepsilon \rightarrow 0$. It was shown in 11, 12] that for the theory on flat space, the definition (4.16) with the boundary conditions (5.1) are in accordance with the conformal normalization (1.3). Since the leading behavior at short distances of the two-point function is not affected by the curvature, the expression (4.16) with the boundary conditions (5.1) lead to the same conformal normalization (1.3) for the theory on the Poincaré disk.

In order to evaluate the limit $\varepsilon \rightarrow 0$ in the expression (4.16), we need the density of angular quantization states $\partial_{\nu} \ln (S(\nu))$, where the S-matrix $S(\nu)$ is associated with the scattering off the "mass barrier" described in the previous section:

$$
\left.\left(\begin{array}{c}
u_{\nu} \\
v_{\nu}
\end{array}\right)\right|_{\eta \rightarrow-\infty}=\left(\begin{array}{c}
e^{i \nu \eta} \\
-i e^{-i \nu \eta} S(\nu)(2 R)^{-2 i \nu}
\end{array}\right)
$$

Here $u_{\nu}$ and $v_{\nu}$ are the partial waves (4.5) and (4.6). The S-matrix is given by:

$$
S(\nu)=(2 R)^{2 i \nu} \frac{\Gamma(1 / 2+i \nu) \Gamma(1 / 2-i \nu+\mu)}{\Gamma(1 / 2-i \nu) \Gamma(1 / 2+i \nu+\mu)} .
$$

Then one finds

$$
\left\langle\mathcal{O}_{\alpha}\right\rangle=\exp \left[\int_{0}^{\infty} \frac{d \nu}{2 \pi i} \ln \left(\frac{\left(1+e^{-2 \pi \nu+2 \pi i \alpha}\right)\left(1+e^{-2 \pi \nu-2 \pi i \alpha}\right)}{\left(1+e^{-2 \pi \nu}\right)^{2}}\right) \partial_{\nu} \ln S(\nu)\right] .
$$

The result of the integration using (5.2) can be expressed in various ways:

$$
\begin{aligned}
\left\langle\mathcal{O}_{\alpha}\right\rangle & =(2 R)^{-\alpha^{2}} \exp \left[\int_{0}^{\infty} \frac{d t}{t}\left(1-e^{-2 \mu t}\right) \frac{\sinh ^{2}(\alpha t)}{\sinh ^{2}(t)}\right] \\
& =(2 R)^{-\alpha^{2}} \prod_{n=1}^{\infty}\left(\frac{1-\frac{\alpha^{2}}{(\mu+n)^{2}}}{1-\frac{\alpha^{2}}{n^{2}}}\right)^{n} \\
& =(2 R)^{-\alpha^{2}} \frac{G(1+\mu-\alpha) G(1+\mu+\alpha)}{G(1+\mu)^{2} G(1-\alpha) G(1+\alpha)},
\end{aligned}
$$

where $G(z)$ is Barnes' $G$-function, characterized mainly by the properties $G(z+1)=\Gamma(z) G(z)$ and $G(1)=1$ (cf. [16]).

\section{Long distance expansion of the two-point function}

As argued in Appendix B.1, the "two-particle" form factors (B.4), like the partial waves (3.5), are entire functions of the spectral parameters $\omega_{1}$ and $\omega_{2}$ (hence all form factors of scaling fields $\mathcal{O}_{\alpha}$ are entire functions of their spectral parameters). Moreover, they have the following behavior as the real part of $\omega_{1}$ or $\omega_{2}$ goes to positive or negative infinity:

$$
\begin{aligned}
& \sqrt{\rho\left(\omega_{1}\right)} F_{\alpha}\left(\omega_{1}, \omega_{2}\right)_{+,-} \sim\left|\omega_{1}\right|^{-1 \pm \alpha} \text { as } \Re e\left(\omega_{1}\right) \rightarrow \pm \infty \\
& \sqrt{\rho\left(\omega_{2}\right)} F_{\alpha}\left(\omega_{1}, \omega_{2}\right)_{+,-} \sim\left|\omega_{2}\right|^{-1 \mp \alpha} \text { as } \Re e\left(\omega_{2}\right) \rightarrow \pm \infty
\end{aligned}
$$

up to proportionality factors, and other "multi-particle" form factors have similar behaviors. Hence for $-1<\alpha-\alpha^{\prime}<1$, the integrals over $\omega_{j}$ 's in (3.11) are absolutely convergent. They can be evaluated by deforming the contours in their lower half planes and summing over the residues at the poles of the 
measure $\rho\left(\omega_{j}\right)$. The measure $\rho(\omega)$ has poles on the imaginary axis; in the lower half plane, they are at positions $\omega=-i(1+2 \mu+2 k)$ for $k \in \mathbb{N}$.

It is instructive to interpret the poles in the measure $\rho(\omega)$, and the evaluation of the integrals in (3.11) by contour deformation as described above, in terms of a different quantization scheme. Let us summarize our construction. We choose an isometry $\mathbf{K}$ (3.1), subgroup of the full isometry group $S U(1,1)$, and we quantize the theory (2.2) on orbits of this isometry. That is, the generator $K$ of the subgroup $\mathbf{K}$ generates "space" translations. A basis for the corresponding Hilbert space $\mathcal{H}$ is taken as a set of states diagonalizing $K$. They are normalized so that all form factors of local fields are entire functions of its eigenvalues $\omega$. With this normalization, the resolution of the identity on $\mathcal{H}$ in terms of this basis involves a measure $\rho(\omega)$ (3.4) with a specific analytical structure, in particular with singularities on the imaginary axis. Now, these singularities should give information about the spectrum in a quantization scheme where $K$ is taken as the Hamiltonian, that is, as the generator of "time" translations.

This last assertion is the analogue of what is well known to happen for instance in a free massive theory on flat space: the singularity structure of the invariant measure $\rho(p)=\left(m^{2}+p^{2}\right)^{-\frac{1}{2}}$ as function of the momentum $p$ gives the energy spectrum. Momentum operator and energy operator (or Hamiltonian) can be seen as representations of the same translation generator in two different quantization scheme, one where this translation is along the "space" direction, the other where it is along the "time" direction.

In a free theory on flat space, the invariant measure exhibits in particular a branch cut starting at $p=-i m$ and going to $p \rightarrow-i \infty$, corresponding to the continuous spectrum (from $m$ to infinity) of the energy operator. In our free theory on the Poincaré disk, the position of the poles of the measure (3.4) on the imaginary axis are interpreted as the discrete eigenvalues of the generator $K$ in the scheme where it is taken as the Hamiltonian. It is a simple matter to repeat the canonical quantization procedure of section 3 for this quantization scheme. One indeed finds a discrete set of eigenstates $\left|k_{1}, \ldots, k_{n}\right\rangle_{\epsilon_{1}, \ldots, \epsilon_{n}}, k_{j} \in$ $\mathbb{N}, n=0,1,2, \ldots$ with eigenvalues $l_{1}+\cdots+l_{n}$, where

$$
l_{j}=1+2 \mu+2 k_{j} .
$$

Since these states diagonalize a generator of time translation, they can be more naturally interpreted as multi-particle states. Matrix elements of the operator $\mathcal{O}_{\alpha}$ between the vacuum $|0\rangle$ and multi-particle states in this scheme can be found from similar matrix elements in the Hilbert space $\mathcal{H}$ by the following identification:

$$
\left\langle 0\left|\mathcal{O}_{\alpha}\right| k_{1}, \ldots, k_{n}\right\rangle_{\epsilon_{1}, \ldots, \epsilon_{n}}=\left\langle\mathcal{O}_{\alpha}\right\rangle \prod_{j=1}^{n}\left(i^{k_{j}} \sqrt{\frac{2 \Gamma\left(1+2 \mu+k_{j}\right)}{k_{j} !}} \frac{1}{\Gamma\left(\frac{1}{2}+\mu\right)}\right) F_{\alpha}\left(-i l_{1}, \ldots,-i l_{n}\right)_{\epsilon_{1}, \ldots, \epsilon_{n}}
$$

and $\epsilon_{1}, \ldots, \epsilon_{n}\left\langle k_{n}, \ldots, k_{1}\left|\mathcal{O}_{\alpha}\right| 0\right\rangle=\left\langle 0\left|\mathcal{O}_{-\alpha}\right| k_{1}, \ldots, k_{n}\right\rangle_{\epsilon_{1}, \ldots, \epsilon_{n}}^{*}$. The resolution of the identity is given by the sum

$$
1=\sum_{n=0}^{\infty} \frac{1}{n !} \sum_{\epsilon_{1}, \ldots, \epsilon_{n}} \sum_{k_{1}, \ldots, k_{n} \geq 0}\left|k_{1}, \ldots, k_{n}\right\rangle_{\epsilon_{1}, \ldots, \epsilon_{n}} \epsilon_{1}, \ldots, \epsilon_{n}\left\langle k_{1}, \ldots, k_{n}\right|,
$$

which provides a long distance expansion for the two-point function:

$$
\begin{aligned}
\left\langle\mathcal{O}_{\alpha}(x) \mathcal{O}_{\alpha^{\prime}}(y)\right\rangle= & \sum_{n=0}^{\infty} \frac{1}{n !} \sum_{\epsilon_{1}, \ldots, \epsilon_{n}} \sum_{k_{1}, \ldots, k_{n}=0}^{\infty} \times \\
& \times\left\langle 0\left|\mathcal{O}_{\alpha}(0)\right| k_{1}, \ldots, k_{n}\right\rangle_{\epsilon_{1}, \ldots, \epsilon_{n}} \epsilon_{n}, \ldots, \epsilon_{1}\left\langle k_{n}, \ldots, k_{1}\left|\mathcal{O}_{\alpha^{\prime}}(0)\right| 0\right\rangle e^{-\left(l_{1}+\cdots+l_{n}\right) \frac{\mathrm{d}(x, y)}{2 R}} .
\end{aligned}
$$

This expansion coincides with the sum of residues coming from the evaluation of the integrals in (3.11) by contour deformation. Using crossing symmetry (4.13) and summing over the $U(1)$ charges, this can be written

$$
\left\langle\mathcal{O}_{\alpha}(x) \mathcal{O}_{\alpha^{\prime}}(y)\right\rangle=\left\langle\mathcal{O}_{\alpha}\right\rangle\left\langle\mathcal{O}_{\alpha^{\prime}}\right\rangle \sum_{N=0}^{\infty} \frac{1}{(N !)^{2}} \sum_{k_{1}, \ldots, k_{2 N}=0}^{\infty} f_{\alpha}\left(k_{1}, \ldots, k_{2 N}\right) f_{\alpha^{\prime}}\left(k_{2 N}, \ldots, k_{1}\right) e^{-\left(l_{1}+\cdots+l_{2 N}\right) \frac{\mathrm{d}(x, y)}{2 R}},
$$


where

$$
\left\langle\mathcal{O}_{\alpha}\right\rangle f_{\alpha}\left(k_{1}, \ldots, k_{2 N}\right)=\left\langle 0\left|\mathcal{O}_{\alpha}(0)\right| k_{1}, \ldots, k_{2 N}\right\rangle_{N}^{+,+, \ldots} \underbrace{-,-, \ldots}_{N} .
$$

The functions $f_{\alpha}\left(k_{1}, \ldots, k_{2 N}\right)$ are evaluated in closed form in Appendices B.2, B.3. The first few terms of (6.1) give the following expansion for the two-point function:

$$
\begin{gathered}
F(t)=1-4^{2 \mu+1} \frac{\sin (\pi \alpha) \sin \left(\pi \alpha^{\prime}\right) \Gamma(1+\mu+\alpha) \Gamma(1+\mu-\alpha) \Gamma\left(1+\mu+\alpha^{\prime}\right) \Gamma\left(1+\mu-\alpha^{\prime}\right)}{\pi^{2} \Gamma(2+2 \mu)^{2}} e^{-(4 \mu+2) t} \times \\
\left(1-2 \alpha \alpha^{\prime} \frac{2 \mu+1}{(\mu+1)^{2}} e^{-2 t}+O\left(e^{-4 t}\right)\right)+O\left(e^{-(8 \mu+4) t}\right),
\end{gathered}
$$

where the function $F(t)$ was defined in (1.5).

It is interesting to notice that in the massless limit $\mu \rightarrow 0$, the two-point function still decreases exponentially at long distance. This is a signal of the infrared regulator properties of the negative curvature of the Poincaré disk [10.

\section{Two-point function from Painlevé VI transcendents}

From 9], the two-point function can be described in terms of a Painlevé VI transcendent:

$$
F(t)=\exp \left[-\int_{\tanh ^{2} t}^{1} d s \frac{d}{d s} \ln \tau(s)\right],
$$

where $\frac{d}{d s} \ln \tau(s)$ is given in (1.2) and the Painlevé transcendent $w(s)$ satisfies (1.1) with $\gamma=0$.

As said in the introduction, the integration constants specifying the Painlevé transcendent can be taken to be the exponents in the behaviors of $w$ at $s \sim 0$ (short distance) and at $s \sim 1$ (long distance), given, respectively, in (1.4) and (1.6) (such a solution exists). The proportionality constants $B$ and $A$ are then unambiguous. In particular, using results of Jimbo [17 there is an explicit expression for the constant $B$ involved in the short distance behavior of $w$ :

$$
B=\mu \frac{\Gamma(\alpha) \Gamma\left(\alpha^{\prime}\right) \Gamma\left(1-\alpha-\alpha^{\prime}\right)^{2} \Gamma\left(\alpha+\alpha^{\prime}+\mu\right)}{\Gamma(1-\alpha) \Gamma\left(1-\alpha^{\prime}\right) \Gamma\left(\alpha+\alpha^{\prime}\right)^{2} \Gamma\left(1-\alpha-\alpha^{\prime}+\mu\right)} .
$$

From this, one can obtain the full short distance expansion of $w$, valid for $0<\alpha+\alpha^{\prime}<1$ :

$$
w=B s^{\alpha+\alpha^{\prime}} \sum_{p, q=0}^{\infty} C_{p, q} s^{p\left(\alpha+\alpha^{\prime}\right)+q\left(1-\alpha-\alpha^{\prime}\right)}
$$

where $C_{0,0}=1$. Once $B$ is fixed, the other coefficients $C_{p, q}$ are uniquely determined by the differential equation (1.1).

The leading long distance asymptotics (1.6) of this Painlevé transcendent is fixed by specifying the proportionality constant $A$. This constant can be obtained by comparing our form factor result (6.3) with the expansion (1.7); this leads to the value (1.8). Since we must have $0<w<1$ for all $0<s<1$, the asymptotics (1.6) is valid only for $A>0$, and the asymptotics (1.4) only for $B>0$. This imposes $\alpha \alpha^{\prime}>0$. From the behavior (1.6) and using the differential equation, one can obtain the full long distance expansion of $w$ :

$$
1-w=A(1-s)^{1+2 \mu} \sum_{p, q=0}^{\infty} D_{p, q}(1-s)^{p(1+2 \mu)+q}
$$

with $D_{0,0}=1$. For instance, the coefficients $D_{0, q}$ are given by

$$
\sum_{q=0}^{\infty} D_{0, q}(1-s)^{q}=s^{\alpha-\alpha^{\prime}} F\left(1-\alpha^{\prime}+\mu, \alpha+\mu ; 1+2 \mu ; 1-s\right)^{2} .
$$


The long distance expansion (7.3) can also be obtained from the full form factors expansion (6.1). For instance, one can verify that the coefficient $D_{0,1}$ in (7.4) is consistent with the coefficient of $e^{-2 t}$ in the parenthesis in the form factor expansion (6.3).

Solving numerically the differential equation by using the appropriate initial conditions, it is possible to verify the consistency of our result (1.8) for the constant involved in the long distance asymptotics of $w$, our result (5.4) for the one-point function, and the value (7.2) for the constant involved in the short distance asymptotics of $w$. We used as initial condition the long distance asymptotics (7.3) with non-zero coefficients $D_{0, q}$ given in (7.4) and with the value (1.8) for the normalization constant. We numerically verified that the behavior (1.4) with the constant (7.2) is recovered, and that the equation (1.3) is satisfied to a high accuracy.

\section{Conclusion}

We have fully characterized the two-point function $\left\langle\mathcal{O}_{\alpha}(x) \mathcal{O}_{\alpha^{\prime}}(y)\right\rangle$ in the Dirac theory on the Poincaré disk, with fermion mass $m$ and Gaussian curvature $-\frac{1}{R^{2}}$, in the region $0<\alpha+\alpha^{\prime}<1, \alpha \alpha^{\prime}>0$ and $m R>\frac{1}{2}$. A comparison of our long distance expansion with the expansion obtained from the Painlevé VI differential equation strongly suggests both that our "form factor" expansion is correct and that the Painlevé VI differential equation indeed describes the two-point function of the scaling fields $\mathcal{O}_{\alpha}$ in the region of parameters above. Notice that our results and the description above in terms of the differential equation are valid in the region $0<m R<\frac{1}{2}$ as well; however, they do not describe all the possible behaviors in this region.

It would be very interesting to extend this description to a larger region of $\alpha, \alpha^{\prime}$, and to fully analyze the region $0<m R<\frac{1}{2}$ and the analytical continuation to negative values of $m R$. Probably one of the most interesting eventual application of this description is to the analysis of an appropriate scaling limit of a "near-critical" lattice Ising model on the Poincaré disk. Two-point functions of flat-space Ising spin and disorder variables in the scaling limit (described by two-point functions of spin and disorder fields in the free Majorana field theory) are simply related to two-point functions of the scaling fields $\mathcal{O}_{ \pm \frac{1}{2}}$ in the free Dirac theory on flat space [6, 7, 8]. If similar relations exist between the Ising spin and disorder variables on the Poincaré disk and the fields $\mathcal{O}_{ \pm \frac{1}{2}}$ in the free Dirac theory on the Poincaré disk, the study of the latter can give information about the effect of the Poincaré geometry on the statistical properties of the Ising model. For instance, the one-point function (5.4) for $\alpha=\frac{1}{2}$ should be proportional to the square of the Ising magnetization near "criticality". In the same spirit, from the point of view of the Ising model on the Poincaré disk, the region $m R<\frac{1}{2}$, and in particular the analytical continuation to negative values of $m R$, should show the most interesting features, especially in view of the arguments of [10. Some work has already been done in relating the scaling fields $\mathcal{O}_{ \pm \frac{1}{2}}$ in the Dirac theory on the Poincaré disk to spin and disorder fields in the Majorana theory on the Poincaré disk [18. We hope to present results of a more complete investigation in a future publication [19].

\section{Acknowledgment}

I am very grateful to S. Lukyanov for suggesting the research project to me and for numerous discussions. I have also extremely benefited from discussions with P. Fonseca, A. B. Zamolodchikov and G. Moore, and comments from C. A. Tracy. This research was supported in part by an NSERC Postgraduate Scholarship. 


\section{A Construction of the embedding}

Here we describe a method for obtaining the expression (4.10) for the operators $a_{\epsilon}(\omega)$. We first solve the locality condition (4.9) for a set of operators $Z_{\epsilon}(r)$ depending on a complex parameter $r$ :

$$
\left\{Z_{\epsilon}(r), \Psi_{\text {pol }}(\eta \rightarrow-\infty)\right\}=\left\{Z_{\epsilon}(r), \Psi_{p o l}^{\dagger}(\eta \rightarrow-\infty)\right\}=0,
$$

with the following ansatz:

$$
Z_{+}(r)=\int_{-\infty}^{\infty} d \nu f(\nu) c_{\nu}^{\dagger} r^{-i \nu}, Z_{-}(r)=\int_{-\infty}^{\infty} d \nu f(\nu) c_{-\nu} r^{-i \nu}
$$

This leads to the equations:

$$
\lim _{\eta \rightarrow-\infty} \int_{-\infty}^{\infty} d \nu e^{i \nu \eta} f(\nu) r^{-i \nu}=0, \lim _{\eta \rightarrow-\infty} \int_{-\infty}^{\infty} d \nu S(\nu)(2 R)^{-2 i \nu} e^{-i \nu \eta} f(\nu) r^{-i \nu}=0,
$$

where $S(\nu)$ is given in (5.2). The first equation is satisfied if $f(\nu)$ is analytical in the lower half $\nu$-plane and increases at most exponentially as $\Im m(\nu) \rightarrow-\infty$, and the second equation is satisfied if $S(\nu) f(\nu)$ is analytical in the upper-half $\nu$-plane and increases at most exponentially as $\Im m(\nu) \rightarrow \infty$. Indeed, under such conditions it is possible, for $\eta$ negative and large enough, to send the contour of integration to $\Im m(\nu) \rightarrow-\infty$ in the integral $\int_{-\infty}^{\infty} d \nu e^{i \nu \eta} f(\nu) r^{-i \nu}$, giving zero contribution, and similarly for the integral $\int_{-\infty}^{\infty} d \nu S(\nu)(2 R)^{-2 i \nu} e^{-i \nu \eta} f(\nu) r^{-i \nu}$ by sending the contour of integration to $\Im m(\nu) \rightarrow \infty$. This set of conditions on $f(\nu)$ forms a simple Riemann-Hilbert problem, a solution of which is:

$$
f(\nu)=\sqrt{2 \pi} \frac{\Gamma\left(\frac{1}{2}+\mu+i \nu\right)}{\Gamma\left(\frac{1}{2}+\mu\right) \Gamma\left(\frac{1}{2}+i \nu\right)} .
$$

The operators $a_{\epsilon}(\omega)$ can then be formed by taking appropriate linear combinations of $Z_{\epsilon}(r)$. These linear combinations can be obtained by requiring that the states $|\omega\rangle_{\epsilon} \equiv a_{\epsilon}(\omega) e^{-\pi H_{A}}$ diagonalize the subgroup $\mathbf{K}$ and that they be correctly normalized. First consider states $|r\rangle_{\epsilon} \in \mathcal{H}$ embedded in $\mathcal{H}_{A} \otimes \mathcal{H}_{A}^{*}$ by the identification $|r\rangle_{\epsilon} \equiv Z_{\epsilon}(r) e^{-\pi H_{A}}$. Using this embedding we can calculate the following matrix elements:

$$
\begin{aligned}
\left\langle v a c\left|\pi_{\mathcal{H}}\left(\Psi_{R}(z, \bar{z})\right)\right| r\right\rangle_{+} & =-i \frac{\Gamma(1+\mu)}{\Gamma\left(\frac{1}{2}+\mu\right)}(1-z \bar{z})^{\mu} r^{-\frac{1}{2}}(1-\bar{z} r)^{-\mu}\left(1-z r^{-1}\right)^{-\mu-1} \\
\left\langle v a c\left|\pi_{\mathcal{H}}\left(\Psi_{L}(z, \bar{z})\right)\right| r\right\rangle_{+} & =\frac{\Gamma(1+\mu)}{\Gamma\left(\frac{1}{2}+\mu\right)}(1-z \bar{z})^{\mu} r^{\frac{1}{2}}\left(1-z r^{-1}\right)^{-\mu}(1-\bar{z} r)^{-\mu-1}
\end{aligned}
$$

From this and from the transformation properties of the fermion fields one can infer the transformation properties of the states $|r\rangle_{\epsilon}$ under the isometry group $S U(1,1)$ :

$$
\hat{g}\left|\frac{a r+\bar{b}}{b r+\bar{a}}\right\rangle_{\epsilon}=H_{\frac{\mu}{2}+\frac{1}{4}, g}(r) \bar{H}_{\frac{\mu}{2}+\frac{1}{4}, g}\left(r^{-1}\right)|r\rangle_{\epsilon},
$$

where the functions $H_{s, g}$ and $\bar{H}_{s, g}$ are automorphic factors:

$$
H_{s, g}(z)=(b z+\bar{a})^{2 s}, \bar{H}_{s, g}(\bar{z})=(\bar{b} \bar{z}+a)^{2 s} .
$$

Diagonalizing this action for the subgroup $\mathbf{K}$, we obtain the operators $a_{\epsilon}(\omega)$ :

$$
a_{\epsilon}(\omega)=\frac{2^{-\mu-\frac{1}{2}} e^{-i \frac{\pi}{2}\left(\mu+\frac{1}{2}+i \frac{\omega}{2}\right)} \Gamma\left(\frac{1}{2}+\mu\right)^{2}}{\sqrt{\pi} \Gamma\left(\frac{1}{2}+\mu+i \frac{\omega}{2}\right) \Gamma\left(\frac{1}{2}+\mu-i \frac{\omega}{2}\right)} \int_{-1}^{1} d r(1-r)^{\mu-\frac{1}{2}+i \frac{\omega}{2}}(1+r)^{\mu-\frac{1}{2}-i \frac{\omega}{2}} r^{-\mu-\frac{1}{2}} Z_{\epsilon}(r),
$$

where the integral is performed in the region $-\pi<\arg (r)<0$ (other integration contours are possible but equivalent). The normalization was fixed by comparing with the partial wave (3.5). The expression (A.3) for $a_{\epsilon}(\omega)$ is equivalent to (4.10). 


\section{B Construction of form factors}

We study the normalized form factors of scaling fields (4.15):

$$
F_{\alpha}\left(\omega_{1}, \ldots, \omega_{n}\right)_{\epsilon_{1}, \ldots \epsilon_{n}}=\left\langle\left\langle a_{\epsilon_{1}}\left(\omega_{1}\right) \cdots a_{\epsilon_{n}}\left(\omega_{n}\right)\right\rangle\right\rangle_{\alpha},
$$

where we use the notation

$$
\langle\langle\cdots\rangle\rangle_{\alpha}=\frac{\operatorname{Tr}\left(e^{-2 \pi K+2 \pi i \alpha Q} \cdots\right)}{\operatorname{Tr}\left(e^{-2 \pi K+2 \pi i \alpha Q}\right)} .
$$

We will assume that the spectral parameters $\omega_{j}$ are real, except when explicitly stated.

\section{B.1 Construction of "two-particle" form factors}

From the cyclic properties of the trace and the anti-commutation relations for the angular quantization modes, we have

$$
\left\langle\left\langle c_{\nu}^{\dagger} c_{\nu^{\prime}}\right\rangle\right\rangle_{\alpha}=\frac{\delta\left(\nu-\nu^{\prime}\right)}{1+e^{2 \pi \nu-2 \pi i \alpha}} .
$$

From the definition (4.10), the trace (4.15) is then expressed as an integral:

$$
\begin{aligned}
& \left\langle\left\langle a_{+}\left(\omega_{1}\right) a_{-}\left(\omega_{2}\right)\right\rangle\right\rangle_{\alpha}=\frac{4^{-\mu} e^{\left(\omega_{2}-\omega_{1}\right) \frac{\pi}{4}}}{\Gamma(1+\mu)^{2}} \int_{-\infty}^{\infty} d \nu \frac{\Gamma\left(\mu+\frac{1}{2}+i \nu\right) \Gamma\left(\mu+\frac{1}{2}-i \nu\right) \cosh (\pi \nu)}{1+e^{2 \pi \nu-2 \pi i \alpha}} \times \\
& F\left(\mu+\frac{1}{2}-i \nu, \mu+\frac{1}{2}-i \frac{\omega_{1}}{2} ; 1+2 \mu ; 2+i 0\right) F\left(\mu+\frac{1}{2}-i \nu, \mu+\frac{1}{2}-i \frac{\omega_{2}}{2} ; 1+2 \mu ; 2-i 0\right) .
\end{aligned}
$$

The contour of integration goes between the poles at $\nu=i \alpha-i / 2$ and $\nu=i \alpha+i / 2$.

The integrand is proportional to $e^{-\pi \nu}(-\nu)^{-1+i\left(\omega_{1}-\omega_{2}\right) / 2}$ when $\nu \rightarrow-\infty$, and to $e^{-\pi \nu}(\nu)^{-1-i\left(\omega_{1}-\omega_{2}\right) / 2}$ when $\nu \rightarrow \infty$. This can be obtained from the asymptotics

$$
F(a, b ; c ; 2 \pm i 0)=\frac{\Gamma(c)}{\Gamma(b)}(2 a)^{b-c} e^{ \pm i \pi(a+b-c)}\left(1+O\left(a^{-1}\right)\right)+\frac{\Gamma(c)}{\Gamma(c-b)}(2 a)^{-b} e^{ \pm i \pi b}\left(1+O\left(a^{-1}\right)\right),
$$

valid for $|a| \rightarrow \infty,|\arg (a)|<\pi$. The integral can be regularized by multiplying the integrand by a factor $e^{p \nu}$ with some complex parameter $p$; the integral is then convergent for $\Re e(p)=\pi, \Im m(p) \neq 0$. It can be evaluated by the method of residues, closing the contour for instance in the upper half plane if $\Im m(p)>0$. The "two-particle" form factor is the analytical continuation to $p=0$ of the resulting expression. Contributions of poles in the upper half plane give

$$
\left\langle\left\langle a_{+}\left(\omega_{1}\right) a_{-}\left(\omega_{2}\right)\right\rangle\right\rangle_{\alpha}=\frac{4^{-\mu} i \pi e^{\left(\omega_{2}-\omega_{1}\right) \frac{\pi}{4}}}{\sin (\pi(\mu-\alpha)) \Gamma(1+\mu)^{2}}\left[G_{\alpha}\left(\omega_{1}, \omega_{2}\right)-e^{-i \pi(\mu-\alpha)} G_{\mu}\left(\omega_{1}, \omega_{2}\right)\right],
$$

where $G_{\alpha}\left(\omega_{1}, \omega_{2}\right)$ is the analytical continuation to $p=0$ of the following series:

$$
\begin{aligned}
& G_{\alpha}^{(p)}\left(\omega_{1}, \omega_{2}\right)=\sum_{n=0}^{\infty}\left[\sin (\pi \alpha) \frac{\Gamma(1+\mu+\alpha+n)}{\Gamma(1-\mu+\alpha+n)} e^{i p\left(\frac{1}{2}+\alpha+n\right)}\right. \\
& \left.\times F\left(\mu+1+\alpha+n, \mu+\frac{1}{2}-i \frac{\omega_{1}}{2} ; 2 \mu+1 ; 2+i 0\right) F\left(\mu+1+\alpha+n, \mu+\frac{1}{2}-i \frac{\omega_{2}}{2} ; 2 \mu+1 ; 2-i 0\right)\right]
\end{aligned}
$$

and $G_{\mu}\left(\omega_{1}, \omega_{2}\right)$ is the analytical continuation to $p=0$ of the series above with $\alpha$ replaced by $\mu$. Note that the function $G_{\mu}\left(\omega_{1}, \omega_{2}\right)$ is shown below to be identically zero.

The series $G_{\alpha}^{(p)}\left(\omega_{1}, \omega_{2}\right)$ is convergent in the upper half $p$-plane $\Im m(p)>0$ as well as on $\Im m(p)=$ $0, \Re e(p) \neq\{0, \pi,-\pi\}$. The analytical continuation to $p=0$ can be done via a "zeta-regularization". 
More precisely, we subtract, inside the summation symbol in $(\underline{B .3})$, the leading large $n$ asymptotics of the summand:

$$
\begin{aligned}
& \Gamma(2 \mu+1)^{2} \sin (\pi \alpha) 4^{-\mu}(2 n)^{-1} e^{\pi \Delta} e^{i p\left(\frac{1}{2}+\alpha+n\right)} \times \\
& {\left[\frac{(2 n)^{-i \omega}}{\Gamma\left(\mu+\frac{1}{2}-i \frac{\omega_{1}}{2}\right) \Gamma\left(\mu+\frac{1}{2}-i \frac{\omega_{2}}{2}\right)}+\frac{(2 n)^{i \omega}}{\Gamma\left(\mu+\frac{1}{2}+i \frac{\omega_{1}}{2}\right) \Gamma\left(\mu+\frac{1}{2}+i \frac{\omega_{2}}{2}\right)}+\right.} \\
& \left.\frac{e^{i \pi(\alpha-\mu+n)}(2 n)^{-i \Delta}}{\Gamma\left(\mu+\frac{1}{2}-i \frac{\omega_{1}}{2}\right) \Gamma\left(\mu+\frac{1}{2}+i \frac{\omega_{2}}{2}\right)}+\frac{e^{-i \pi(\alpha-\mu+n)}(2 n)^{i \Delta}}{\Gamma\left(\mu+\frac{1}{2}+i \frac{\omega_{1}}{2}\right) \Gamma\left(\mu+\frac{1}{2}-i \frac{\omega_{2}}{2}\right)}\right]
\end{aligned}
$$

where $\omega=\left(\omega_{1}+\omega_{2}\right) / 2$ and $\Delta=\left(\omega_{1}-\omega_{2}\right) / 2$. The resulting series is convergent at $p=0$. We then add to this series at $p=0$ the following quantity:

$$
\begin{aligned}
& \Gamma(2 \mu+1)^{2} \sin (\pi \alpha) 4^{-\mu} e^{\pi \Delta} \times \\
& {\left[\frac{1}{\Gamma\left(\mu+\frac{1}{2}-i \frac{\omega_{1}}{2}\right) \Gamma\left(\mu+\frac{1}{2}-i \frac{\omega_{2}}{2}\right)} \frac{\zeta(1+i \omega)}{2^{1+i \omega}}+\frac{1}{\Gamma\left(\mu+\frac{1}{2}+i \frac{\omega_{1}}{2}\right) \Gamma\left(\mu+\frac{1}{2}+i \frac{\omega_{2}}{2}\right)} \frac{\zeta(1-i \omega)}{2^{1-i \omega}}+\right.} \\
& \left.\frac{e^{i \pi(\alpha-\mu)}\left(2^{-i \Delta}-1\right)}{\Gamma\left(\mu+\frac{1}{2}-i \frac{\omega_{1}}{2}\right) \Gamma\left(\mu+\frac{1}{2}+i \frac{\omega_{2}}{2}\right)} \frac{\zeta(1+i \Delta)}{2^{1+i \Delta}}+\frac{e^{-i \pi(\alpha-\mu)}\left(2^{i \Delta}-1\right)}{\Gamma\left(\mu+\frac{1}{2}+i \frac{\omega_{1}}{2}\right) \Gamma\left(\mu+\frac{1}{2}-i \frac{\omega_{2}}{2}\right)} \frac{\zeta(1-i \Delta)}{2^{1-i \Delta}}\right],
\end{aligned}
$$

where $\zeta(z)$ is Riemann's zeta function. The result is $G_{\alpha}\left(\omega_{1}, \omega_{2}\right)$. Notice that the function $G_{\alpha}\left(\omega_{1}, \omega_{2}\right)$ is real for real $\omega_{1}, \omega_{2}$.

The resulting expression for $G_{\alpha}\left(\omega_{1}, \omega_{2}\right)$ defines a function of $\omega_{1}$ and $\omega_{2}$ analytical in the region $\left|\Im m\left(\omega_{1}+\omega_{2}\right)\right|<2,\left|\Im m\left(\omega_{1}-\omega_{2}\right)\right|<2$. By repeating the procedure above for the full large $n$ asymptotics of the summand of $G_{\alpha}^{(p)}\left(\omega_{1}, \omega_{2}\right)$, one can see that the function $G_{\alpha}\left(\omega_{1}, \omega_{2}\right)$ thus defined has no singularity in the finite complex $\omega_{1}$ - and $\omega_{2}$-planes: the function $G_{\alpha}\left(\omega_{1}, \omega_{2}\right)$ is an entire function of $\omega_{1}$ and $\omega_{2}$.

The function $G_{\mu}\left(\omega_{1}, \omega_{2}\right)$ can now be evaluated in the following way. From (B.2), we have

$$
\left\langle\left\langle a_{+}\left(\omega_{1}\right) a_{-}\left(\omega_{2}\right)\right\rangle\right\rangle_{0}=-\frac{4^{-\mu} i \pi e^{\left(\omega_{2}-\omega_{1}\right) \frac{\pi}{4}}}{\sin (\pi \mu) \Gamma(1+\mu)^{2}} e^{-i \pi \mu} G_{\mu}\left(\omega_{1}, \omega_{2}\right) .
$$

By covariance under the subgroup $\mathbf{K}$ we find

$$
\left(\omega_{1}+\omega_{2}\right) G_{\mu}\left(\omega_{1}, \omega_{2}\right)=0 .
$$

Since the function $G_{\mu}\left(\omega_{1}, \omega_{2}\right)$ is entire, this implies that it must be identically zero. Notice that a similar calculation leads to $\left\langle\left\langle a_{-}\left(\omega_{1}\right) a_{+}\left(\omega_{2}\right)\right\rangle\right\rangle_{0}=0$. Since the commutator $\left\{a_{-}\left(\omega_{1}\right), a_{+}\left(\omega_{2}\right)\right\}$ is a $c$-number, an immediate consequence is the anti-commutation property (4.11).

Hence we finally have

$$
F_{\alpha}\left(\omega_{1}, \omega_{2}\right)_{+,-}=\frac{4^{-\mu} i \pi e^{\left(\omega_{2}-\omega_{1}\right) \frac{\pi}{4}}}{\sin (\pi(\mu-\alpha)) \Gamma(1+\mu)^{2}} G_{\alpha}\left(\omega_{1}, \omega_{2}\right) .
$$

\section{B.2 Two-particle form factors in the discrete basis}

It is possible to evaluate the function $G_{\alpha}\left(\omega_{1}, \omega_{2}\right)$ for some purely imaginary values of $\omega_{1}$ and $\omega_{2}$. We will evaluate it for the values $\omega_{1}=-i\left(1+2 \mu+2 k_{1}\right)$ and $\omega_{2}=-i\left(1+2 \mu+2 k_{2}\right)$ for integers $k_{1} \geq 0$ and $k_{2} \geq 0$. This can be done by using the analytical continuation described above. Equivalently, it can be done by simply replacing these values of $\omega_{1}$ and $\omega_{2}$ in the expression (B.3.3), evaluating the resulting series at $p=0$ in a region of $\mu$ where it is convergent and analytically continuing the result in $\mu$. With

$$
G_{\alpha ; k_{1}, k_{2}} \equiv G_{\alpha}\left(-i\left(1+2 \mu+2 k_{1}\right),-i\left(1+2 \mu+2 k_{2}\right)\right),
$$


this gives

$$
G_{\alpha ; k_{1}, k_{2}}=\sin (\pi \alpha) \sum_{m_{1}=0}^{k_{1}} \sum_{m_{2}=0}^{k_{2}} \frac{\left(-k_{1}\right)_{m_{1}}\left(-k_{2}\right)_{m_{2}} 2^{m_{1}+m_{2}}}{(2 \mu+1)_{m_{1}}(2 \mu+1)_{m_{2}} m_{1} ! m_{2} !} H_{\alpha ; m_{1}, m_{2}}
$$

where

$$
H_{\alpha ; m_{1}, m_{2}}=\frac{\Gamma\left(1+\mu+\alpha+m_{1}\right) \Gamma\left(1+\mu+\alpha+m_{2}\right)}{\Gamma(1-\mu+\alpha) \Gamma(1+\mu+\alpha)}{ }_{3} F_{2}\left(1,1+\mu+\alpha+m_{1}, 1+\mu+\alpha+m_{2} ; 1+\mu+\alpha, 1-\mu+\alpha ; 1\right) .
$$

The ${ }_{3} F_{2}$ hypergeometric function above can be evaluated in closed form, for any given integer $m_{1}$ and $m_{2}$, in terms of Gamma functions and rational functions of $\mu$ and $\alpha$. The two-particle form factors (6.2) in the discrete basis are expressed in terms of $G_{\alpha ; k_{1}, k_{2}}$ :

$$
f_{\alpha}\left(k_{1}, k_{2}\right)=2^{2 \mu+1} i(-1)^{k_{1}} \sqrt{\frac{\Gamma\left(1+2 \mu+k_{1}\right) \Gamma\left(1+2 \mu+k_{2}\right)}{k_{1} ! k_{2} !}} \frac{G_{\alpha ; k_{1}, k_{2}}}{\Gamma(1+2 \mu)^{2} \sin (\pi(\mu-\alpha))} .
$$

\section{B.3 "Multi-particle" form factors}

The "multi-particle" form factors of scaling fields can be evaluated by Wick's theorem in terms of the two-particle form factors. We have

$$
F_{\alpha}\left(\omega_{1}, \ldots, \omega_{n}\right)_{\epsilon_{1}, \ldots, \epsilon_{n}}=\sum_{j=2}^{n}(-1)^{j} F_{\alpha}\left(\omega_{1}, \omega_{j}\right)_{\epsilon_{1}, \epsilon_{j}} F_{\alpha}\left(\omega_{2}, \ldots, \widehat{\omega_{j}}, \ldots, \omega_{n}\right)_{\epsilon_{2}, \ldots, \widehat{\epsilon_{j}}, \ldots, \epsilon_{n}}
$$

where the hat over an argument means omission of this argument. Of course this will be non-zero only for $\sum_{j=1}^{n} \epsilon_{j}=0$. Forming the $n \times n$ matrix $\mathbf{F}_{\alpha}\left(\omega_{1}, \ldots, \omega_{n}\right)_{\epsilon_{1}, \ldots, \epsilon_{n}}$ with matrix elements $\left[\mathbf{F}_{\alpha}\left(\omega_{1}, \ldots, \omega_{n}\right)_{\epsilon_{1}, \ldots, \epsilon_{n}}\right]_{i, j}=$ $F_{\alpha}\left(\omega_{i}, \omega_{j}\right)_{\epsilon_{i}, \epsilon_{j}}$, the "multi-particle" form factors can be written as Pfaffians"

$$
F_{\alpha}\left(\omega_{1}, \ldots, \omega_{n}\right)_{\epsilon_{1}, \ldots, \epsilon_{n}}=\operatorname{Pf}\left(\mathbf{F}_{\alpha}\left(\omega_{1}, \ldots, \omega_{n}\right)_{\epsilon_{1}, \ldots, \epsilon_{n}}\right) .
$$

Using (4.11), it is always possible to choose the order such that $N$ operators with positive $U(1)$ charge are followed by $N$ operators with negative $U(1)$ charge. Forming the $N \times N$ matrix $\mathbf{G}_{\alpha}\left(\omega_{1}, \ldots, \omega_{N} ; \tilde{\omega}_{1}, \ldots \tilde{\omega}_{N}\right)$ with matrix elements $\left[\mathbf{G}_{\alpha}\left(\omega_{1}, \ldots, \omega_{N} ; \tilde{\omega}_{1}, \ldots \tilde{\omega}_{N}\right)\right]_{i, j}=F_{\alpha}\left(\omega_{i}, \tilde{\omega}_{j}\right)_{+-}$, we have

$$
F_{\alpha}\left(\omega_{1}, \ldots, \omega_{N}, \tilde{\omega}_{1}, \ldots, \tilde{\omega}_{N}\right) \underbrace{+,+, \ldots}_{N} \underbrace{-,-, \ldots}_{N}=(-1)^{N(N-1) / 2} \operatorname{det}\left(\mathbf{G}_{\alpha}\left(\omega_{1}, \ldots, \omega_{N} ; \tilde{\omega}_{1}, \ldots, \tilde{\omega}_{N}\right)\right) \text {. }
$$

Similar expressions are valid for the form factors $\left\langle 0\left|\mathcal{O}_{\alpha}\right| k_{1}, \ldots, k_{n}\right\rangle_{\epsilon_{1}, \ldots, \epsilon_{n}}$ in the discrete basis.

\section{Flat space limit of form factors}

It is a simple matter to verify that the "two-particle" form factors (B.4) specialize to the known expression in the flat space limit. Two-particle form factors of the scaling fields $\mathcal{O}_{\alpha}$ in the flat space limit, with particle at rapidities $\beta_{1}$ and $\beta_{2}$ and states normalized by ${ }_{\epsilon_{1}}\left\langle\beta_{1} \mid \beta_{2}\right\rangle_{\epsilon_{2}}^{\text {flat }}=2 \pi \delta_{\epsilon_{1}, \epsilon_{2}} \delta\left(\beta_{1}-\beta_{2}\right)$, are obtained by

$$
\begin{aligned}
& \left\langle\text { vac }\left|\mathcal{O}_{\alpha}\right| \beta_{1}, \beta_{2}\right\rangle_{+-}^{\text {flat }}=\lim _{\mu \rightarrow \infty} \times \\
& (2 \mu)^{\alpha^{2}+1} 2 \pi \sqrt{\rho\left(2 \mu \sinh \left(\beta_{1}\right)\right) \rho\left(2 \mu \sinh \left(\beta_{2}\right)\right) \cosh \left(\beta_{1}\right) \cosh \left(\beta_{2}\right)} F_{\alpha}\left(2 \mu \sinh \left(\beta_{1}\right), 2 \mu \sinh \left(\beta_{2}\right)\right)_{+-}
\end{aligned}
$$

and the one-point function is obtained by

$$
\left\langle\mathcal{O}_{\alpha}\right\rangle^{\text {flat }}=\lim _{\mu \rightarrow \infty}(2 \mu)^{\alpha^{2}}\left\langle\mathcal{O}_{\alpha}\right\rangle
$$

\footnotetext{
${ }^{1}$ Using this, one can immediately write the form factor expansion 3.11 as a Fredholm determinant in the case $\alpha=\alpha^{\prime}$.
} 
Using the expression (B.4), this gives the known normalized flat space form factors, first obtained in [7]:

$$
\frac{\left\langle\text { vac }\left|\mathcal{O}_{\alpha}\right| \beta_{1}, \beta_{2}\right\rangle_{+-}^{\text {flat }}}{\left\langle\mathcal{O}_{\alpha}\right\rangle^{\text {flat }}}=\frac{i \sin (\pi \alpha)}{\cosh \left(\frac{\beta_{1}-\beta_{2}}{2}\right)} e^{\alpha\left(\beta_{1}-\beta_{2}\right)} .
$$

\section{References}

[1] N. B. Birrel and P. C. W. Davies, Quantum Fields in Curved Space, Cambridge University Press, Cambridge, New York (1982)

[2] M. Sato, T. Miwa and M. Jimbo, Holonomic quantum fields, Publ. RIMS, Kyoto University, 15 (1979) III: pp. 577-629, IV: pp. 871-972

[3] M. Jimbo, T. Miwa and K. Ueno, Monodromy preserving deformation of linear ordinary differential equations with rational coefficients. I. General theory and $\tau$-function, Physica 2D (1981) pp. 306-352

[4] M. Jimbo and T. Miwa, Monodromy preserving deformation of linear ordinary differential equations with rational coefficients. II, Physica 2D (1981) pp. 407-448

[5] J. Palmer, Determinants of Cauchy-Riemann operators as $\tau$-functions, Acta Applicandae Mathematicae 18 (1990) pp. 199-223

[6] J. B. Zuber and C. Itzykson, Quantum field theory and the two-dimensional Ising model, Phys. Rev. D15 (1977) pp. 2875-2884

[7] B. Schroer and T. T. Truong, The order/disorder quantum field operators associated with the twodimensional Ising model in the continuum limit, Nucl. Phys. B144 (1978) pp. 80-122

[8] T. T. Wu, B. M. McCoy, C. A. Tracy and E. Barouch, Spin-spin correlation functions for the twodimensional Ising model: exact theory in the scaling region, Phys. Rev. B13 (1976) pp. 316-374

[9] J. Palmer, M. Beatty and C. A. Tracy, Tau functions for the Dirac operator on the poincaré disk, Commun. Math. Phys. 165 (1994) pp. 97-174, hep-th/9309017

[10] C. Callan and F. Wilczek, Infrared behavior at negative curvature Nucl. Phys. B340 (1990) pp. $366-386$

[11] Al. B. Zamolodchikov, unpublished

[12] S. Lukuyanov and A. B. Zamolodchikov, Exact expectation values of local fields in quantum sineGordon model, Nucl. Phys. B493 (1997) pp. 571-587 hep-th/9611238

[13] S. Lukyanov, Free field representation for massive integrable models, Commun. Math. Phys. 167 (1995) pp. 183-226, hep-th/9307196

[14] V. Brazhnikov and S. Lukyanov, Angular quantization and form-factors in massive integrable models, Nucl. Phys. B512 (1998) pp. 616-636, hep-th/9707091

[15] S. Khoroshkin, A. Leclair and S. Pakuliak, Angular quantization of the sine-Gordon model at the free fermion point, Adv. Theor. Math. Phys. 3 (1999) pp.1227-1287 hep-th/9904082

[16] H. M. Srivastava and J. Choi Series Associated with the Zeta and Related Functions, Kluwer Academic Publishers, Dordrecht, Boston, London (2001)

[17] M. Jimbo, Monodromy problem and the boundary condition for some Painlevé equations, Publ. RIMS, Kyoto University, 18 (1982) pp. 1137-1161 
[18] B. Doyon, Form factor of Ising spin and disorder fields on the Poincaré disk, J. Phys. A37 (2003) pp. 359-370, special edition, proceedings of the workshop Recent Advances in the Theory of Integrable Systems (Annecy, 2003).

[19] B. Doyon, P. Fonseca, work in progress. 